\title{
Translational theranostic methodology for diagnostic imaging and the concomitant treatment of malignant solid tumors
}

\author{
Hemant Sarin
}

\begin{abstract}
Pathologic hyperpermeability exists in the spectrum of disease states, including neuro-ischemic, neuro-inflammatory and neuro-oncological. To-date the characterization of disease pathology with $T_{1}$-weighted quantitative dynamic contrast-enhanced magnetic resonance imaging has relied on the study of modeled microvascular parameters such as diseased tissue transvascular flow rates of small molecule paramagnetic imaging agents with short plasma half-lives, based on which it is difficult to assess the specific nature of the disease state. Another type of $\mathrm{T}_{1}$-weighted quantitative dynamic contrast-enhanced magnetic resonance imaging involves the use of paramagnetic heavy metal-chelated dendrimer nanoparticles that possess longer plasma half-lives with pre-determined molar relaxivities to quantitatively image concentration of macromolecular contrast agent accumulation over time in hyperpermeable pathology such as solid tumor tissue with the important advantage of concomitantly treating the pathology, which, in recent years, has been shown to be possible with the use of optimally-sized theranostic dendrimer nanoparticles in the 7 to 10 nanometer size range that are functionalized biocompatibly with a small molecule therapeutic. In this focused review, this translational theranostic methodology for quantitative dynamic contrast-enhanced magnetic resonance imaging and the concomitant treatment of malignant solid tumors with optimally designed theranostic nanoparticles within the 7 to 10 nanometer size range is discussed in context of fine-tuning its suitability for the study and treatment of other disease states with pathologic hyperpermeability and without.
\end{abstract}

Keywords: Nanoparticle, Functionalized dendrimer, Quantitative magnetic resonance imaging, Bi-compartmental modeling, Intravenous administration, Drug delivery, Blood half-life, Pathologic angiogenesis, Primary brain tumor, Metastases

\section{Introduction}

Pathologic angiogenesis is a hallmark of hyperpermeable disease states of chromic hypoxia, particularly solid malignancies, irrespective of type or grade, with there being a physiologic upper limit of pore size greater than that of normal healthy tissue blood capillaries with few exceptions [1-3]. In other disease states of hyperpermeability such as neuro-ischemic disease the character of microvascular barrier dysfunction depends on the time course of hypoxia, whereby acute ischemia results in cytotoxic edema and in secondarily altered diffusion of water molecules within the tissue interstitia [4] followed by varying periods of endothelial cell dysfunction and compromised inter-

Correspondence: hsmd74@hotmail.com

${ }^{1}$ Charleston, West Virginia (WV), USA endothelial junctional complex integrity of varying degrees [5], as may be the case in the hyperpermeability of neuroinflammatory disease.

Diagnostic imaging with hydrophilic contrast agents enables the detection of pathologic hyperpermeability in the form of abnormal contrast enhancement of the diseased tissue interstitium. This abnormal contrast enhancement of diseased tissue is observable following the intravenous infusion of hydrophilic small molecule contrast agents but requires administration at relatively high doses, of tens of grams of the hydrophilic small molecule contrast agent, and often necessitates the intra-arterial or intra-thecal administration due to the non-selectivity of these contrast agents combined with their relatively short plasma halflives, which is irrespective of imaging modality, whether 
it is computed tomography (CT), positron emission tomography (PET) or magnetic resonance imaging (MRI). Furthermore, due to the sub-optimal pharmacokineticodyamics of small molecule contrast agents and the requirement for the administration of high doses, the issue of systemic toxicity arises and is not uncommon, which can manifests as systemic hypersensitivity, either as immediate or delayed, depending on the biophysical properties of the contrast agent, whereby contrast agents such as Omnipaque 370, an iodinated hexol for CT, can cause immediate-type hypersensitivity reactions, while those such as gadopentetate dimeglumine (Magnevist) or gadodiamide (Omniscan), both being poorly chelated forms of polyvalent heavy metal Gadolinium $(\mathrm{Gd} 3+)$ cause delayed-type hypersensitivity [6], in contrast to when $\mathrm{Gd} 3+$ is introduced into the biological system in its much more stably chelated biocompatible cyclic form of Gd-DOTA (Gd-Gadoteric acid) [7].

Although the mainstay of present day diagnostic neurovascular magnetic resonance imaging in everyday clinical practice remains the acquisition of images in signal space without respect to the quantification of relative contrast enhancement [8], magnetic resonance imaging affords the ability to detect contrast-enhanced signal change in real-time at exquisite resolution as compared to CT or PET imaging, whereby contrast-enhanced signal change can be quantified accurately for MRI contrast agents of appropriate exterior biophysical character $[3,9]$. Of the select premier academic centers that offer dynamic contrast-enhanced MRI-based quantification, these centers offer bi-compartmental model-based estimates of permeability surface area product parameters such as Ktrans (forward transvascular flow rate per min; permeability surface area product) [10-12] and $\mathrm{V} p$ (vascular plasma volume) [12], parameters derived on the basis of the bi-compartmental modeling of change in normalized contrast-enhanced plasma signal to that of the change in normalized contrast-enhanced diseased tissue signal over time with contrast agents having short blood half-lives [10,12,13]. Even as model-derived parameters identify areas of hyperpermeability consistent with pathologic hyperpermeability [14] and offer perspective into therapeutic efficacy for pathologies of known etiology [15], these parameters such as Ktrans are also normally elevated for hyperpermeable healthy tissues [16,17], being non-specific parameters, making it difficult to discriminate solid tumor tissue from normally hyperpermeable tissues such as muscle tissue [18], especially at tumor infiltrating border zones where the permeability surface area product values are marginally elevated $[16,18]$.

The poor diagnostic specificity of dynamic contrastenhanced MRI-based bi-compartmentally modeled microvascular permeability parameters of small molecule contrast agent pharmacokineticodynamics [19-23] cannot be overcome by temporarily increasing the blood half-life of a small molecule contrast agent by decreasing its renal filtration fraction [12,24], or alternatively, by increasing the dose, due to the non-selective extravasation of small molecule contrast agents of less than 2 nanometers into healthy tissues with loose macula occludens junctions or fenestrated endothelial cells $[1,2]$. The poor diagnostic cum therapeutic specificity of small molecule contrast agent pharmacokineticodynamics, however, can be improved by the utilization of biocompatible macromolecular agents of the dendritic sub-class of larger sizes within the optimal size range of 7 to 10 nanometers $[3,8,25,26]$ because even as the per minute forward transvascular flow rate (Ktrans) from blood plasma into the solid tumor interstitium decreases significantly with increasing contrast agent molecular size [27,28], the blood plasma half-life of a contrast agent increases with increasing size, whereby the contrast agent's blood plasma half life becomes the primary determinant of the extensiveness of intratumoral contrast agent accumulation within solid tumor tissue $[3,8,28,29]$, which becomes important for the diagnostic imaging, and importantly, the treatment of solid tumors, in which small molecule chemotherapeutics do not build up to effective concentrations [3,30,31].

The current status of the field of translational theranostics is reviewed herein in context of a methodological discussion of the prototypical quantitative dynamic contrastenhanced magnetic resonance imaging-based theranostic approach for the concomitant diagnosis and non-invasive treatment of solid tumors, one which has translational potential towards clinical application.

\section{Review}

Small molecules and nanoparticles for non- and semi-quantitative diagnostic imaging

Small molecules with biophysical properties of specific character have applications for a variety of diagnostic imaging modalities including dense atom-based for CT imaging, radioisotope-based for PET imaging, photostable luminescent atom- or fluorescent organic biomoleculebased for fluorescent imaging, as well as paramagnetic atom-based such as Gadolinium-based for magnetic resonance imaging [32], however, they possess short blood plasma half-lives and lack polyvalency, in contrast to their commensurate macromolecular nanoparticulate-based forms.

Due to the limitations of small molecule-based diagnostic imaging probes, particularly short blood plasma half-lives and lack polyvalency, over decades, macromolecular and polymeric nanoparticle forms have been developed, including for quantum dot-based fluorescent imaging for in vivo applications [33,34], and those of greater probability for biocompatibility investigated for clinical imaging, including for PET imaging, which include 
radioisotopes conjugated to $150 \mathrm{kDa}$ antibodies that possess longer blood half-lives at a hydrodynamic molecular size of approximately 12 nanometers [35], as well as for diagnostic MRI for the clinical setting, which include: (1) iron oxide core-based ferromagnetic nanoparticles with various types of non-covalent dextran-based coatings, beginning in earlier years with interest in the clinical diagnostic imaging application of individual particles in the 16 to 35 nanometer size range [36-38], and cumulating in most recent years towards the clinical development of very small citrated-dextran coated iron oxide nanoparticles (VSOPs) with an anionic exterior and a molecular size of approximately 8 nanometers for macrophage imaging due to the propensity for de-coating and the uptake of iron oxide cores by cells of the monocyctemacrophage phagocytic system [39]; and (2) a dendritic conjugate of chelated paramagnetic heavy metal Gadolinium, Gadomer-17, a $17 \mathrm{kDa}$ dendrimer-based nanoparticle with anionic exterior polyvalency and an improved blood half-life at a molecular size of approximately 3 nanometers, which has been utilized exclusively as a blood pool contrast agent for perfusion magnetic resonance imaging [40].

In addition to Gadomer-17, other spherical monodisperse nanoparticles of the dendrimer sub-class have been extensively studied in more recent decades, for their applications as blood pool agents for blood and lymphatic perfusion MRI [41-43], as well as for studying microvascular permeability of hyperpermeable pathology [43-45], which include the most biocompatible of the imageable dendrimer sub-class, those employing cyclic tetracarboxylic acid DOTA as the chelate for Gadolinium, and demonstrate biocompatible biophysical character including exterior functionalization, shape and size, happening to fall within the molecular size range of $2 \mathrm{~nm}$ to $14 \mathrm{~nm}$ : This being the case, however, not much emphasis has been placed on the following two crucial aspects the first being on the applicability of the imageable dendrimer sub-class for therapeutic purposes including those in the optimal size range for selective transvascular drug delivery of chemotherapy into hyperpermeable pathologies such as solid tumors, and the second being, on the applicability of dynamic contrast-enhanced imaging such as dynamic contrastenhanced MRI to non-invasively quantify therapeutic delivery into hyperpermeable pathologies voxel-by-voxel, until most recently $[1,3,8,25,26,28,29]$.

In the few years from 2006 to 2009, it was determined that imageable dendrimer-based theranostic nanoparticles that possess longer blood plasma half-lives (Figures 1 and 2) achieve effective concentrations in pathologic tissue cells of solid tumors $[3,8,28,29]$, with the optimal size range for nanoparticles with an anionically neutralized exterior being between 7 and 10 nanometers in hydrodynamic diameter $[3,8,25]$, which is due to solid

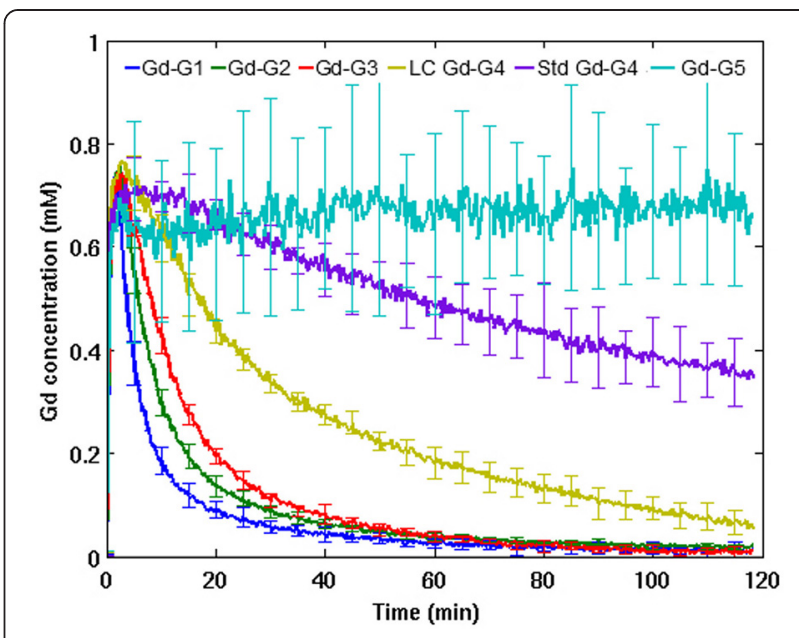

Figure 1 Superior sagittal sinus blood plasma Gd concentration curves over 2 hours following 1 minute bolus intravenous infusion of Gd-DTPA dendrimer generations G1 through G8 infusions at dose of $0.09 \mathrm{mmol} \mathrm{Gd} / \mathbf{k g}$ body weight (bw). Blood plasma concentrations measured via dynamic contrast-enhanced MRI-based analysis by selection of 5-to-10 voxels in the superior sagittal sinus (SSS) free from partial volume averaging effects. Gd-G1 $(n=5), G d-G 2(n=6), G d-G 3(n=6)$, lowly conjugated $(L C) G d-G 4$ $(n=4)$, standard (Std) Gd-G4 ( $(n=6), G d-G 5(n=6), G d-G 6(n=5)$, Gd-G7 $(n=5)$ and Gd-G8 $(n=6)$. Gd-G5 through Gd-G8 dendrimers rapidly reach and maintain a steady state in blood over 2 hours (G6, G7 and G8 not shown for clarity). Error bars represent standard deviation (s.d.) and are shown once every five minutes for clarity. Adapted from references $[3,8,28]$.

tumor blood capillary microvasculature having a physiologic upper limit of pore size of approximately 12 nanometers [29], it being more defective than the blood capillary microvasculature supplying normal healthy tissues [1]. During the course of this experimental studies, the sensitivity of a prototypical translational theranostic approach for quantifiable dynamic contrast-enhanced imaging-based concomitant imaging and treatment of solid malignancies has been validated in over 150 rodent subjects bearing over 250 orthotopic and ectopic malignant gliomas from volumes beginning at $20 \mathrm{~mm}^{3}$ (2-3 $\mathrm{mm}$ diameter tumors) utilizing 7.5 nanometer diameter-sized Gadolinium-DTPAdendrimer nanoparticles infused over 1 minute $[28,29]$ (Figures 3, 4, 5, 6, 7; Table 1) and 9 nanometer diametersized Gadolinium-DTPA-dendrimer-Doxorubicin nanoparticles infused intravenously over 2 minutes [3,8] (Figure 8; Table 1).

Historical overview on the development of nanoparticles for therapeutic purposes: From liposomes to dendrimers Beginning in the 1980s, there has been a virtual explosion in the field of polymeric science for biological applications [46-49], which has led to the development of various investigational macromolecular therapeutics as vehicles for the delivery of small molecule chemotherapeutics based 

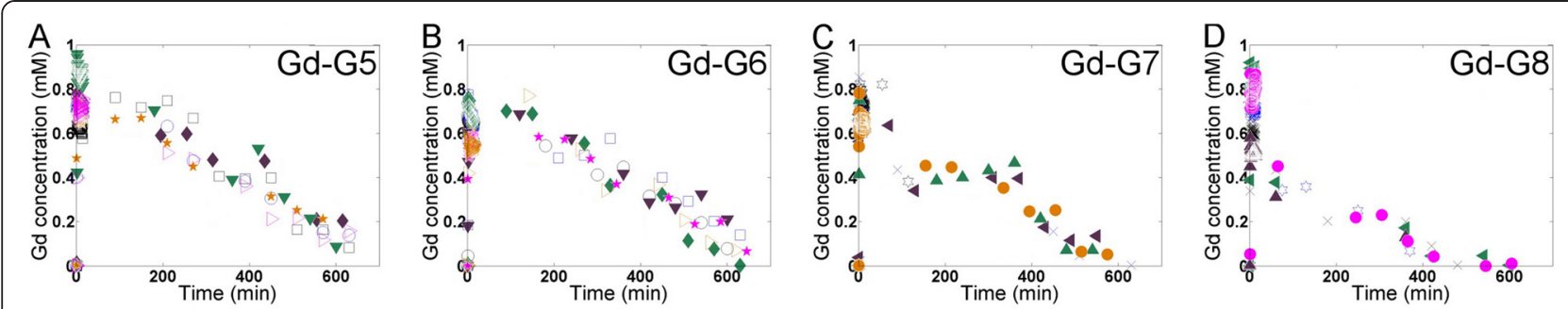

Figure 2 Common carotid artery blood plasma Gd concentration curves over approximately 10 hours following 1 minute bolus intravenous infusion of Gd-DTPA dendrimer generations G5 through G8 at doses of $0.09 \mathbf{~ m m o l ~ G d / k g ~ b o d y ~ w e i g h t ~ ( b w ) . ~ E s t i m a t i o n ~ o f ~}$ theranostic nanoparticle blood half-lives are determined via DC-E MRI-based analysis by the selection of 5-to-10 voxels free from blood flow and partial volume averaging effects in a blood vessel of large caliber, the common carotid artery (CCA). Of note, different sets of 5-to10 voxels selected for each time point with censoring of aberrant $T_{1}$ values as necessary. Rodent subject n: Gd-G5 $(n=6)$, Gd-G6 $(n=6)$, Gd-G7 $(n=5)$ and Gd-G8 ( $n=5)$. Adapted from reference [29]. A) Gd-G5, B) Gd-G6, C) Gd-G7, D) Gd-G8.

on the enhanced permeation and retention (EPR) phenomenon [47,50] including to overcome multi-drug resistance [51] via local sustained release [52], but without emphasis on the optimal size range or design for biocompatibility in the biological system in the physiologic state, until recently $[1-3,7,8,25,26]$. However, it has since then been realized that more attention must be paid to the biophysical properties of macromolecular therapeutics such as the purposeful introduction of cationicity to the exterior of sub-optimally sized nanoparticles in order to improve their permeation across the blood-tumor barrier and diffusion through the tumor interstitium [53,54], which results in significant non-selective toxicity to the biological system in the physiologic state $[2,3,25,26]$. Of the selected subset of these macromolecular drug carriers that have been tested in the clinical setting for therapeutic applications, these include liposomes in the 100 to 200 nanometer size range $[55,56]$; it is sufficient to reiterate that for non-pegylated liposomal doxorubicin (Myocet/Doxil), there is the rapid uptake of the non-pegylated forms (Myocet/Doxil) by the monocycte-macrophage phagocytic and reticuloendothelial systems, which results in only micormolar concentrations in blood plasma [57], and that for the pegylated forms (Caelyx) with longer circulatory half lives than that of the non-pegylated form [57], there is a widespread myoinflammatory response manifesting as different grades of palmar-plantar erythrodysesthesia (hand-foot syndrome) [58], which is likely a form of microvascular inflammatory necrosis (author observation) due to the continuous slow release of low concentrations of doxorubicin, a cationic lipophile anthracycline antibiotic of hydro-lipophilic character, which is directly toxic to cellular membranes, particularly to capillary wall endothelial cell membranes: Importantly, in the cases of

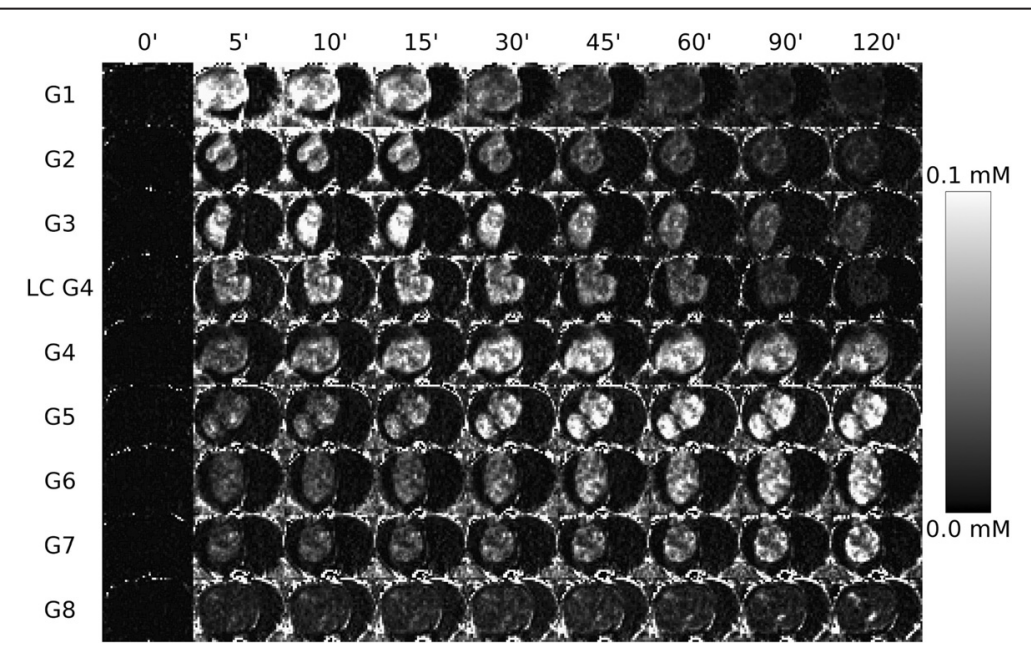

Figure $3 T_{1}$-weighted dynamic contrast-enhanced MRI voxel-by-voxel Gd concentration maps of Gd-dendrimer distribution over time within large orthotopic RG-2 malignant brain tumors. The volume, in $\mathrm{mm}^{3}$, for each large orthotopic RG-2 glioma: Gd-G1, 104; Gd-G2, 94; Gd-G3, 94; lowly conjugated (LC) Gd-G4, 162; standard Gd-G4, 200; Gd-G5, 230; Gd-G6, 201; Gd-G7, 170; and Gd-G8, 289. Respective Gd-dendrimer generations administered intravenously over 1 minute at a Gd dose of $0.09 \mathrm{mmol} \mathrm{Gd} / \mathrm{kg}$ animal body weight. Scale from $0 \mathrm{mM}[\mathrm{Gd}]$ to $0.1 \mathrm{mM}[\mathrm{Gd}]$. Adapted from references $[3,8,28]$. 


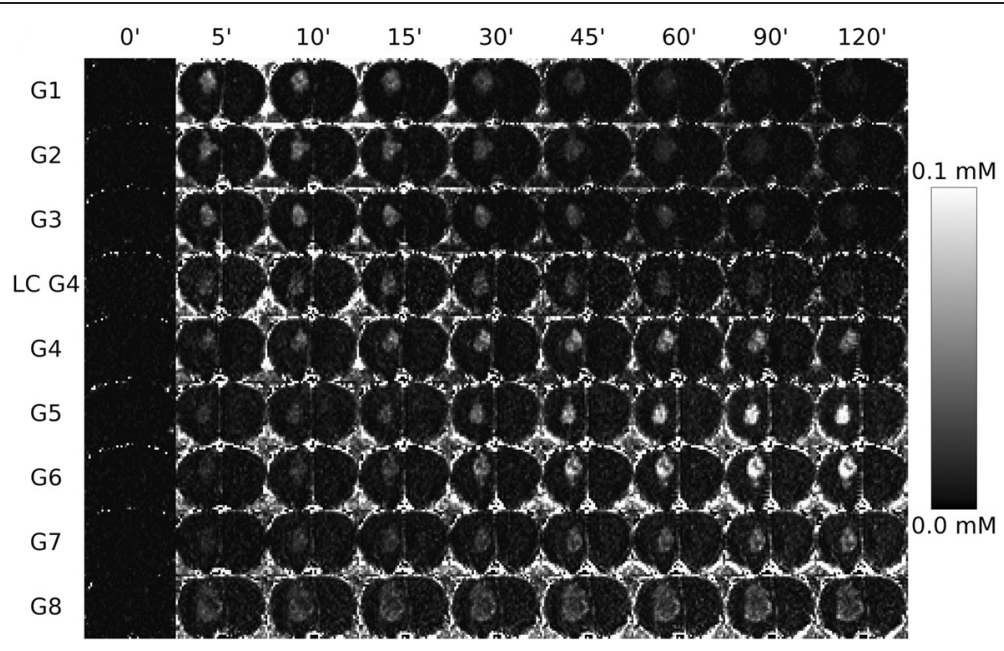

Figure $4 T_{1}$-weighted dynamic contrast-enhanced MRI Gd voxel-by-voxel Gd concentration maps of Gd-dendrimer distribution over time within small orthotopic RG-2 malignant brain tumors. The volume, in $\mathrm{mm}^{3}$, for each small orthotopic RG-2 glioma: Gd-G1, 27; Gd-G2, 28; Gd-G3, 19; lowly conjugated (LC) Gd-G4, 24; standard Gd-G4, 17; Gd-G5, 18; Gd-G6, 22; Gd-G7, 24; and Gd-G8, 107. Respective Gd-dendrimer generations administered intravenously over 1 minute at a $\mathrm{Gd}$ dose of $0.09 \mathrm{mmol} \mathrm{Gd} / \mathrm{kg}$ animal body weight. Scale from $0 \mathrm{mM}[\mathrm{Gd}]$ to $0.1 \mathrm{mM}$ [Gd]. Adapted from references [3,8,28].

both, there is the release of small molecule free drug is into systemic circulation, whereby the free drug itself does not accumulate to effective concentrations within solid tumor tissue itself, due to a upper limit of pore size of approximately 12 nanometers in the hyperpermeable blood capillaries supplying solid tumor tissue $[28,29]$.

In more recent years, there has been exquisite interest in the development of various kinds of biocompatible nanoparticles for therapeutic purposes that remain in the optimal size range of 7 to 10 nanometers upon functionalization, but which has been tempered by the realization that it is difficult to engineer such biocompatibly-sized nanoparticles having covalently-bound stable biocompatible exteriors without toxic metal cores that do not biodegrade. Examples of nanoparticles in the optimal size range that fail to meet this criterion include the 8 nanometer-sized very small Iron Oxide core nanoparticles with a non-covalently-bound dextran organic biomolecule coating (VSOP-C184) [59], as well as the 5 to 10 nanometer-sized Cadmium-Tellurium or CesiumSelenium core quantum dots, which have non-biocompatible metal cores and require non-covalent capping with ZincSulfur and coated with organic biomolecules that themselves may or may not be biocompatible [33].

In contrast to other nanoparticles within the optimal size range, dendrimers are unique nanoparticles [8,25], as dendrimers are spherical nanoparticles with optimal terminal branch surface area-to-volume ratios and no significant increase in volume upon hydration, with the naked, non-functionalized, dendrimer being a repeating biocompatible monomeric units with terminal branches increasing exponentially by $2^{x}$ units doubling for each successive full-generation, beginning with reference to the dendritic core, the zero generation dendrimer (G0) having 4 terminal branches $(x=2)$, with the $1^{\text {st }}$ generation dendrimer (G1: $1.4 \mathrm{kDa} ; 1.9 \mathrm{~nm}$ diameter [Dendritic Nanotechnologies, Inc]) having 8 terminal branches $(x=4)$ and the $5^{\text {th }}$ generation dendrimer (G5: $29 \mathrm{kDa}$; Diameter est at $5.5 \mathrm{~nm}[60])$ having 128 terminal branches $(\mathrm{x}=7)$ $(\mathrm{NH} 3+)$, for example, whereas half-generation dendrimers (G1.5, G2.5 etc.) have intermediate numbers of terminal branches (COO-, $\mathrm{OH})[61,62]$. Even though dendrimer-based nanoparticles have been extensively researched in the past for their potential to be effective at chemotherapeutic drug delivery into solid tumors [63-69], including for folate receptor-targeted chemotherapeutic drug delivery [70-74], these investigations have been without emphasis on the optimal size range of 7 to 10 nanometers, the optimal density or the optimal exterior functionalization for effective, passively selective transvascular delivery, without risk of systemic toxicity, biophysical aspects that have important translational implications $[1-3,7,8,25,26]$ (Table 1).

Optimally sized and designed MRI imageable functionalized dendrimers for theranostic purposes

Nanoparticles of the dendrimer class are monodisperse [60], and functionalized MRI imageable dendrimers with anionically neutralized exteriors maintain their monodispersity within a very narrow size distribution in their respective generation as determined by transmission electron microscopy $[28,29,75]$, whereby the higher generation dendrimers functionalized with paramagnetic heavy metal 

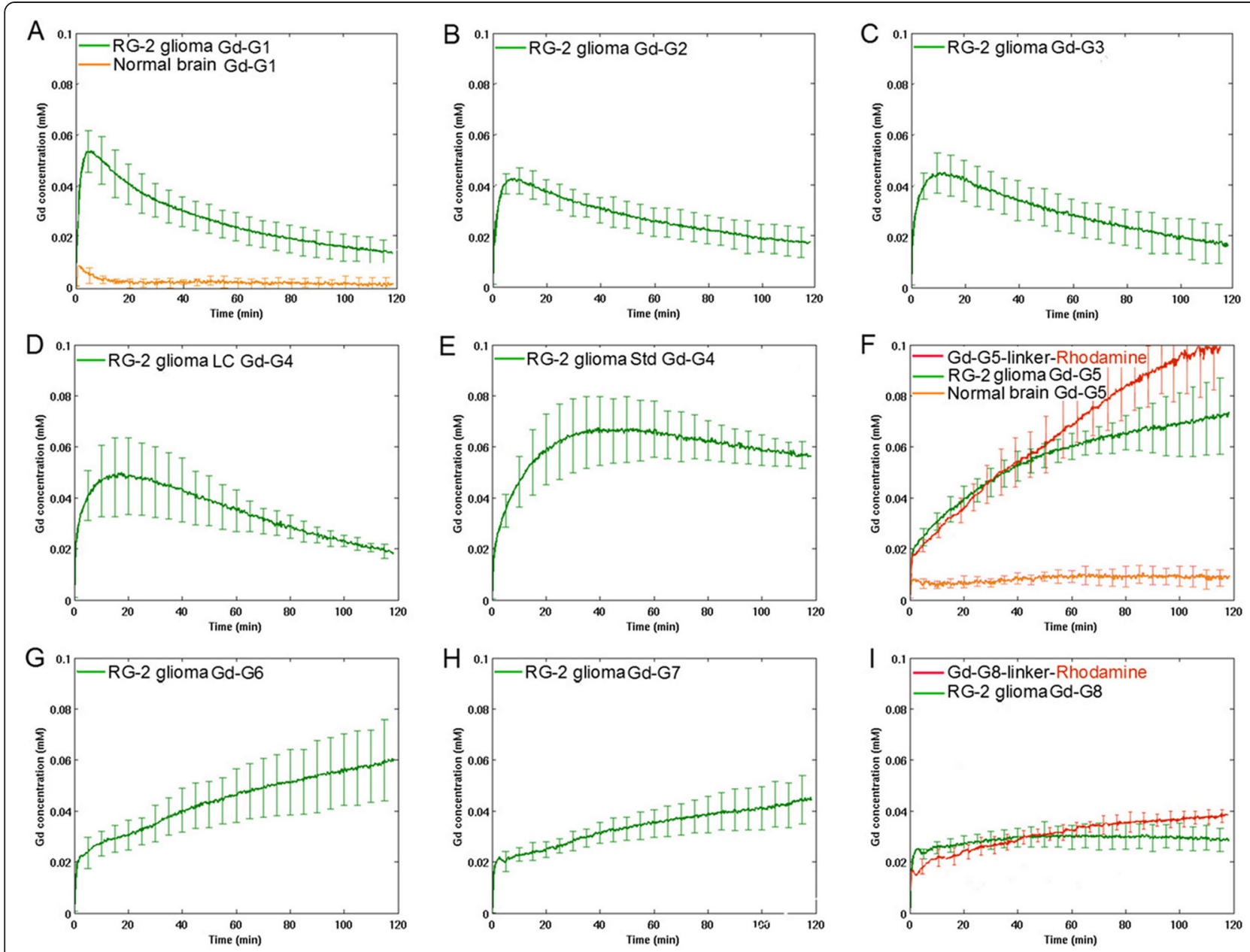

Figure 5 Orthotopic RG-2 malignant glioma tumor tissue Gd concentration curves over 2 hours upon 1 minute bolus intravenous infusion of Gd-DTPA dendrimer generations at dose of $0.09 \mathrm{mmol} \mathrm{Gd} / \mathrm{kg}$ bw and infusion of Gd-DTPA dendrimer-linker-Rhodamine dendrimer generations at dose of $\mathbf{0 . 0 6} \mathbf{~ m m o l ~ G d / k g ~ b o d y ~ w e i g h t ~ ( b w ) . ~ R G - 2 ~ g l i o m a ~ t u m o r ~ t i s s u e ~ c o n c e n t r a t i o n ~ c u r v e s ~ a r e ~ w e i g h t e d ~ w i t h ~}$ respect to total tumor volume (large anterior and small posterior contralateral) with error bars representing standard deviation (s.d.) and shown once every five minutes for clarity. Tumor N per group: Gd-G1 $(n=10), G d-G 2(n=12), G d-G 3(n=12)$, lowly conjugated (LC) Gd-G4 $(n=8)$, standard (Std) Gd-G4 ( $n=12), G d-G 5(n=12)$, Gd-G6 ( $n=10)$, Gd-G7 ( $n=10)$ and Gd-G8 ( $n=12)$; Gd-G5-linker-Rhodamine $(n=10)$ and Gd-G8-linker-Rhodamine $(n=4)$. Of note, Normal brain tissue curves concentration curves for Gd-G1 and Gd-G5 dendrimer groups are shown of $9 \mathrm{~mm}^{3}$ brain volumes, which reflect intravascular blood concentration of Gd-dendrimer Gd in the absence of any extra-vascular extravasation of the respective Gd-dendrimer generations (other Gd-dendrimer generations not shown for clarity). Based on the tumor tissue extravasation concentration curves of the non-cationotoxic Gd-dendrimers (non-Rhodamine functionalized), the upper limit of pore size of the blood-tumor barrier is between the size of the Gd-G7 dendrimers ( 11 nm) and that of the Gd-G8 dendrimers ( 13 nm). Of note, Gd-G5 dendrimer-linker-Rhodamine and Gd-G8 dendrimer-linker-Rhodamine tumor tissue concentration curves accumulate over time in tumor tissue at 2/3 the dose of the non-Rhodamine Gd-dendrimers, the Gd-G5 dendrimer-linker-Rhodamine dendrimer to a significant extent, which is due to cationic toxicity of the blood-tumor barrier, which is also the case of the Gd-G8 dendrimer-linker-Rhodamine (Curve not shown: The cationotoxic extravasation across the normal blood brain-barrier and accumulation in normal brain tissue, which reflects cationic toxicity to the normal healthy blood-brain barrier) Adapted from references [3,8,28]. A) Gd-G1, B) Gd-G2, C) Gd-G3, D) LC Gd-G4, E) Std Gd-G4, F) Gd-G5, G) Gd-G6, H) Gd-G7, I) Gd-G8.

chelates on the exterior remain within the 7 to 10 nanometer size range following functionalization, which is the case for the range of Gd-DTPA-G5 dendrimer (80$85 \mathrm{kDa}$; Diameter est at $7.5 \mathrm{~nm}$ ) [3,8,28,29,76] (Table 1). In the case of the Gd-DTPA-G5 dendrimer-linker-Doxorubicin $(85 \mathrm{kDa})$, its diameter as measured by dynamic light scattering in serum albumin protein-free solution, is at 9 nanometers [3] (Table 1), due to the linker attachment of
Doxorubicin to the exterior, which protrudes $\sim 1.5$ nanometers above the nanoparticles surface.

In the case of MRI imageable functionalized dendrimers, it is notable that there is a significant increase in the density of nanoparticle molecular weight upon the conjugation of chelated paramagnetic heavy metal Gadolinium $(0.157 \mathrm{kDa})$ either by DTPA $(0.393 \mathrm{kDa})$ or by DOTA $(0.404 \mathrm{kDa})$ as when $\sim 50 \%$ of the terminal amines of 


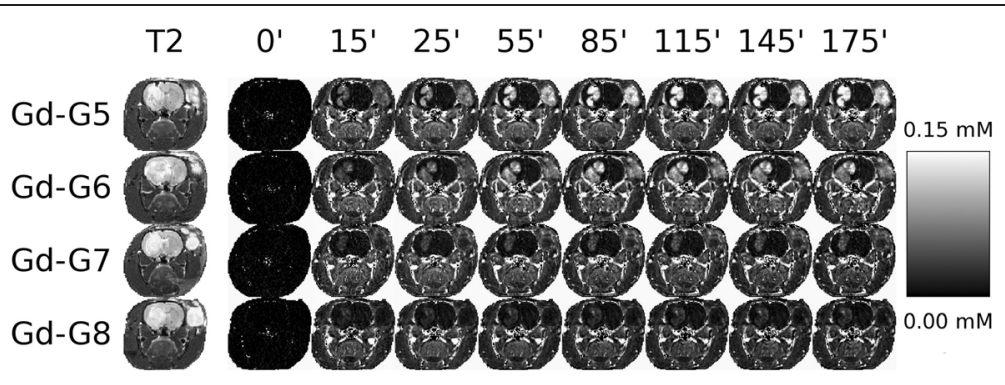

Figure $6 T_{2}$-weighted anatomical MRI images and $T_{1}$-weighted dynamic contrast-enhanced MRI voxel-by-voxel Gd concentration maps of higher generation Gd-dendrimer distribution over approximately 3 hours within orthotopic and ectopic RG-2 malignant solid tumors. The volume, in $\mathrm{mm}^{3}$, for each orthotopic and ectopic RG-2 glioma: First row, Gd-G5; $45 \mathrm{~mm}^{3}$ (brain), $113 \mathrm{~mm}^{3}$ (peripheral); Second row, Gd-G6; $97 \mathrm{~mm}^{3}$ (brain), $184 \mathrm{~mm}^{3}$ (peripheral); Third row, Gd-G7; $53 \mathrm{~mm}^{3}$ (brain), $135 \mathrm{~mm}^{3}$ (peripheral); Fourth row, Gd-G8; 50 mm³ (brain); $163 \mathrm{~mm}^{3}$ (peripheral). Respective Gd-dendrimer generations administered intravenously over 1 minute at a Gd dose of $0.09 \mathrm{mmol} \mathrm{Gd} / \mathrm{kg}$ animal body weight. Scale from $0.00 \mathrm{mM}[\mathrm{Gd}]$ to $0.15 \mathrm{mM}[\mathrm{Gd}]$. Adapted from references $[8,29]$.

naked polyamidoamine dendrimer generation 5 (G5) are conjugated the cationic exterior of the naked polyamidoamine dendrimer (Table 1), it is anionically neutralized due to the presence of 2- charges of the Gd-DTPA/DOTA chelates protruding on the exterior of the MRI imageable functionalized dendrimer. Furthermore, the Gd-DTPA functionalized molecular weight is $\sim 2.6$-fold that of the naked dendrimer weight (Table 1), and importantly, the functionalized dendrimer size is only $\sim 1.5$ nanometers larger than that of the naked dendrimer (Table 1), which is superior to method of exterior pegylation to increase molecular weight in which case the overall size of the molecule increases disproportionately to greater than that of the 7 to 10 nanometer size range. Therefore, imageable heavy metal chelate-based functionalization helps maintain a compact configuration that becomes important for optimally prolonged blood plasma half life as the densely chelated compact nanoparticle does not proteolytically degrade as rapidly in systemic circulation, which has an anionically neutralized exterior.

The other important consideration with regards to the optimal overall design of MRI imageable functionalized theranostic dendrimer nanoparticles within the 7 to 10 nanometer size range towards clinical application is the biophysical character of the functionalized nanoparticle exterior upon outermost exterior covalent functionalization of small molecule probes with inherent lipophilic cationicity such as fluorescent dye, linker-Rhodamine or
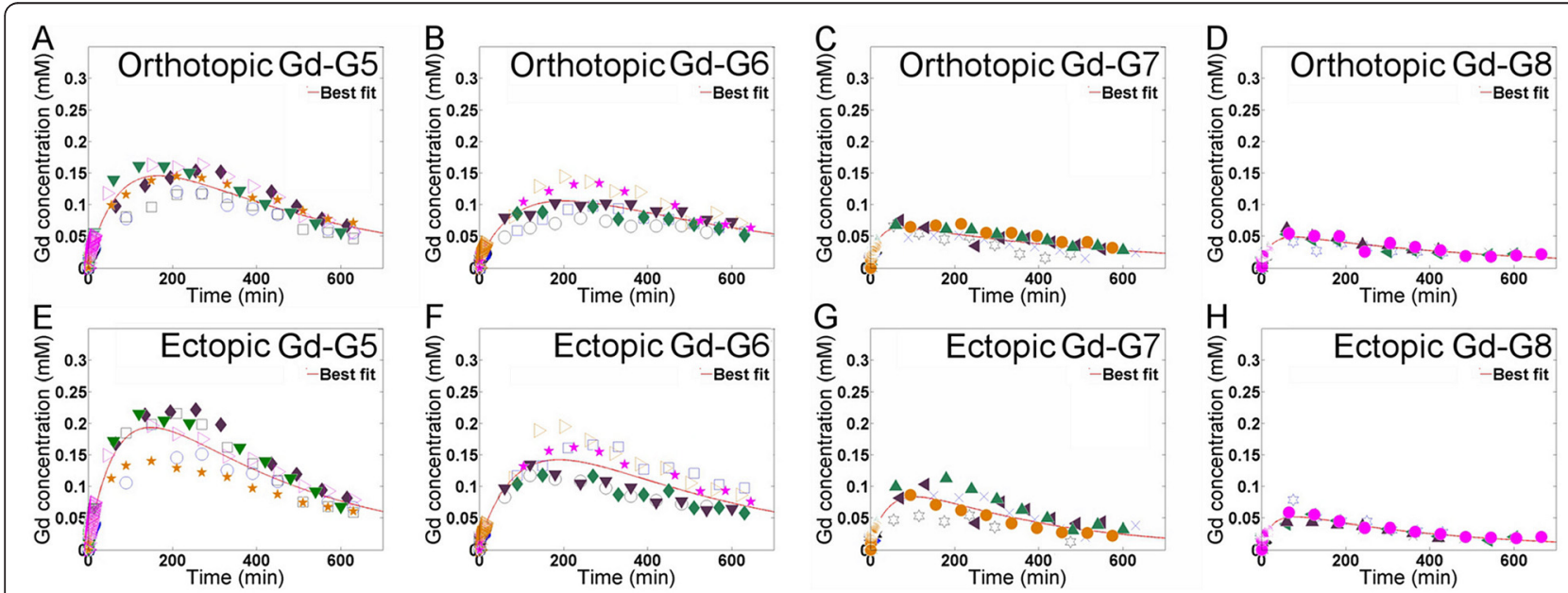

Figure 7 Orthotopic and ectopic RG-2 malignant glioma tumor tissue Gd concentration cures over 10 hours following 1 minute bolus intravenous infusion of Gd-DTPA dendrimer generations G5 through $\mathbf{G} 8$ at doses of 0.09 mmol Gd/kg body weight (bw). Orthotopic and ectopic RG-2 malignant solid tumor tissue concentration curves of intra-cranial caudate nucleus tumors (average volume $\sim 100 \mathrm{~mm}^{3}$ ) and contralateral extra-cranial temporalis muscle tumors (average volume $\sim 150 \mathrm{~mm}^{3}$ ) grown in the same rodent subjects. The $\mathrm{Gd}$ concentration values of free moving rodent subjects in-between scans represent whole tumor average $\mathrm{Gd}$ concentrations determined on the basis of co-registered tumor slice volume region-of-interest values for entire tumor volumes and not voxel-by-voxel. Shown is the best fit curve by least squares minimization. Orthotopic n: $G d-G 5(n=6), G d-G 6(n=6), G d-G 7(n=5)$ and $G d-G 8$ $(n=5)$; Ectopic n: Gd-G5 ( $(n=6), G d-G 6(n=6), G d-G 7(n=5)$ and $G d-G 8(n=5)$. Adapted from reference [8,29]. A) Gd-G5 Orthotopic RG-2 tumor, B) $G d-G 6$ Orthotopic RG-2 tumor, C) Gd-G7 Orthotopic RG-2 tumor, D) Gd-G8 Orthotopic RG-2 tumor, E) Gd-G5 Ectopic RG-2 tumor, F) Gd-G6 Ectopic RG-2 tumor, G) Gd-G7 Ectopic RG-2 tumor, H) Gd-G8 Ectopic RG-2 tumor. 
Table 1 Biophysical properties of naked dendrimers and functionalized dendrimer-based theranostic nanoparticles

\begin{tabular}{|c|c|c|c|c|c|c|c|c|c|}
\hline $\begin{array}{l}\text { Polyamidoamine } \\
\text { dendrimer }(G)\end{array}$ & $\begin{array}{l}\text { Terminal } \\
\text { groups (\#) }\end{array}$ & $\begin{array}{l}\text { Naked } \\
\text { dendrimer } \\
\text { weight (kDa) }\end{array}$ & $\begin{array}{l}\text { Naked } \\
\text { dendrimer } \\
\text { size }(\mathrm{nm}) \&\end{array}$ & $\begin{array}{l}\text { Gd-DTPA } \\
\text { conjugation } \\
(\%) \sim 1\end{array}$ & $\begin{array}{l}\text { Small } \\
\text { molecule drug } \\
\text { conjugation }(\%) \sim 2\end{array}$ & $\begin{array}{l}\text { Funtionalized } \\
\text { dendrimer } \\
\text { weight }(\mathrm{kDa})^{\wedge}\end{array}$ & $\begin{array}{l}\text { Gd-DTPA } \\
\text { dendrimer } \\
\text { size }(\mathrm{nm})\end{array}$ & $\begin{array}{l}\text { Theranostic } \\
\text { dedrimer } \\
\text { size }(\mathrm{nm})^{*}\end{array}$ & $\begin{array}{l}\text { Molar } \\
\text { relaxivity } \\
\text { (r:ms-1/mM) }\end{array}$ \\
\hline Gt-dtpa-G1 & 8 & 1.43 & 1.9 & 67.1 & & 5.63 & & & 9.8 \\
\hline Gt-dtpa-G2 & 16 & 3.26 & 2.6 & 65.9 & & 11.2 & & & 10.1 \\
\hline Gt-dtpa-G3 & 32 & 6.91 & 3.6 & 47.7 & & 18.6 & & & 10.4 \\
\hline Low Gd-dtpa-G4 & 64 & 14.2 & 4.4 & 29.8 & & 24.4 & & & 7.8 \\
\hline Std Gd-dtpa-G4 & 64 & 14.2 & 5.7 & 47.5 & & 39.8 & & & 12.2 \\
\hline Gd-dtpa-G5 & 128 & 28.8 & est 5.5 & 47.2 & & 79.8 & est 7.5 & & 109 \\
\hline Theranostic G5 & 128 & 28.8 & est 5.5 & 48.1 & 7.8 & 85.0 & $\mathrm{n} / \mathrm{a}$ & 9 & 10.1 \\
\hline Gd-dtpa-G6 & 256 & 58.0 & 6.9 & 39.9 & & 133 & est 10 & & 10.6 \\
\hline Gd-dtpa-G7 & 512 & 116 & 8.0 & 50 & & $330 \wedge$ & 11 & & 10.3 \\
\hline Gd-dtpa-G8 & 1024 & 233 & 10.3 & 37.8 & & $597 \wedge$ & 13 & & 9.4 \\
\hline
\end{tabular}

Blank = not available.

$\mathrm{G}=$ Dendrimer Genaration.

$\&=$ Combination of outside data from Dynamic light scattering and TEM findings of Ref 60 and Dendritic Nanotechnologies,Inc.

$\sim 1$ = Based on (MALDI TOF Functionalized Dendrimer Weight - Naked Dendrimer Weight) / 725; Gd-DTPA unit Weight = 725 Daltons.

Doxorubicin (544 Da) + Linker Weight (278 Da) $=822$ Daltons (8 Doxorubicin per Funtionalized Dendrimer).

* = Dynamic light scattering measurement for Gd-DTPA-dendrimer-Hydrazone LINKER-Doxorubicin; Otherwise estimated or measured per ADF TEM.

$\wedge=$ Measured by ADF TEM; Otherwise measured by MADLI TOF spectroscopy.

chemotherapeutic antibiotic, linker-Doxorubicin, which is a form of hydro-lipophilicity [7]: Nanoparticles with cationic exteriors are particularly toxic to cellular membranes, due to the presence of multivalent exterior cationicity protruding outwards from the nanoparticle (Figure 5, Panels F and I; Figure 8), which, when present on the exterior of circulating nanoparticles cause systemic blood-endothelial cell capillary barrier damage as evidenced by non-selective contrast enhancement of the normal blood-brain barrier on dynamic contrast-enhanced MRI $[3,8]$; this is the case for the prototype of a MRI imageable theranostic dendrimer, whose biophysical character is altered due to the introduction of cationic lipophilicity onto its surface that protrudes above the biocompatible anionicity of its preexisting chelated exterior $[2,3,7,8]$, which results in nonselective systemic toxicity to normal healthy microvascular barriers including to the normal blood-brain barrier $[3,8]$ (Figure 8) and is the likely reason for the observed dose-limiting systemic toxicities in experimental studies with dendrimer-based therapeutics with targeting or therapeutic moieties of cationic lipophilic character on the exterior [72,77-79].

It can therefore be surmised that dendrimers are truly unique nanoparticles in that they possess the inherent inner architecture to proteolytically biodegrade along with the potential to be functionalized on the exterior via covalent modifications whereby their biophysical properties can be fine-tuned for: (1) Optimizing size to within the 7 to 10 nanometer range $[1-3,8,25,26]$, which is optimal for passively selective transvascular delivery into hyperpermeable pathologies such as solid tumors but not to healthy tissues [2] (Figures 3 through 7; Table 1); (2) Optimizing density by covalent attachment of strongly chelated imageable heavy metals $[3,8,25,26]$, which maintains their compactness and slows proteolytic degradation while in systemic blood circulation [2,7] (Figures 1 and 2); and (3)

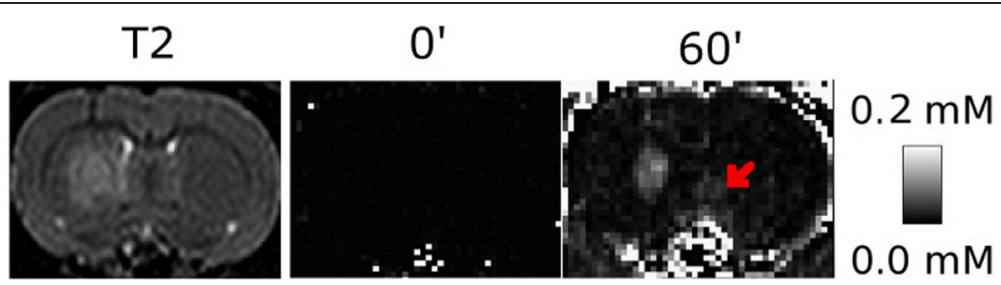

Figure $8 T_{2}$-weighted anatomic MRI scan image of an orthotopic RG-2 malignant brain tumor and $T_{1}$-weighted dynamic contrast-enhanced MRI voxel-by-voxel Gd concentration maps at baseline and at 60 minutes following Gd-G5-doxorubicin dendrimer infusion. Red arrow highlights non-selective contrast enhancement in normal brain tissue. The Gd-G5-doxorubicin dendrimer administered intravenously over 2 minutes at a Gd dose of $0.09 \mathrm{mmol} \mathrm{Gd} / \mathrm{kg}$ animal body weight, which is equivalent to a doxorubicin dose of $8 \mathrm{mg} / \mathrm{kg}$ animal body weight. Scale from $0 \mathrm{mM}$ [Gd] to $0.2 \mathrm{mM}[\mathrm{Gd}]$. Adapted from reference [3,8]. 
Optimizing exterior functionality for anionicity or hydroxylated anionicity via covalent attachment $[3,8,25,26]$, which makes them biocompatible in blood circulation for prolonged duration and maximizes their blood half-life while rendering them free from biophysical interactions with circulatory or interstitial proteins such as serum albumins [2,7] (Figures 1 and 2).

\section{Quantitative dynamic contrast-enhanced imaging for theranostic purpose}

In the case of contrast-enhanced CT- and PET-based imaging employing purely hydrophilic contrast agents there is a linear correlation between the observed contrast enhancement signal and concentration of the contrast agent in tissue as these modalities detect either the opacity or the radioopacity, respectively, of the contrast agent. This, however, is not the case for contrast-enhanced MRI where the observed contrast enhancement signal is a function of several factors that must be taken into consideration the concentration of the agent infused and the temperature, the imaging sequence repletion and echo times $\left(\mathrm{T}_{R}, \mathrm{~T}_{E}\right)$ and the amount of the decrease in the longitudinal relaxation time $\left(\mathrm{T}_{1}\right)$ and increase in longitudinal relaxation rate $\left(\mathrm{R}_{1}, 1 / \mathrm{ms}\right)$ of water molecule protons resulting per unit concentration of the contrast agent $\left(r, \mathrm{~ms}^{-1} / \mathrm{mM}\right)$ in context of the Larmor precession frequency $(\omega)$, which is a product of the gyromagnetic ratio $(\mathrm{g})$ of a charged protons of water molecules and the strength of the magnetic field $\left(B_{0}\right)$ [80]. In magnetic resonance imaging, tissue signal results when energy is applied (E) when a radiofrequency pulse is applied in a static magnetic field to perturb tissue water molecule protons (nuclear spin with $1+$ charge) already precessing at the Larmor frequency in alignment with the magnetic field ( $\mathrm{M}$ vector: $M_{0}$, Equilibrium Magnetization), with the rate of re-alignment being detected at the sequence pre-determined time of detection (Echo time: $T_{\mathrm{E}}$ ) by a receiving coil. Repetitive firing of radiofrequency pulses (Repetition time: $\mathrm{T}_{R}$ ) of a predetermined strength (flip angle $\alpha$ ) results in repetitive deviations from $\mathrm{z}$-axis ( $\mathrm{Mz}$ longitudinal axis vector) towards the $\mathrm{x}-\mathrm{y}$ plane (Mx-My plane vectors) that are measured during the return of the longitudinal magnetization (longitudinal re-growth time: $\mathrm{T}_{1}$ relaxation time), which is the basis for the $\mathrm{T}_{1}$-weighted MRI image generation following Fourier transforms of the detected signal, the $\mathrm{T}_{1}$ signal.

In dynamic contrast-enhanced imaging, a small molecule or macromolecular contrast agent is infused while images are acquired sequentially in real-time, these being radiographic images in the case of dynamic $\mathrm{CT}$ and PET, and resonance images in the case of dynamic MRI, making it possible to observe the difference in the baseline pre-contrast signal to that of the contrast-enhanced signal (normalized signal), which, in the case of dynamic contrast-enhanced MRI can be further quantified in terms of contrast agent concentration by applying the dual-flip angle methodology and the pre-determined molar relaxivity of a hydrophilic contrast agent $\left(r: \mathrm{ms}^{-1} / \mathrm{mM}\right)$, upon which concentration curves and maps can be generated voxel-by-voxel for the duration of imaging session (All figures except Figure 7). In the case of purely hydrophilic small molecule contrast agents such as Gd-DTPA/ DOTA $(0.73 \mathrm{kDa} ; 0.8 \mathrm{~nm})$, which has an overall charge of 2-, a temporal resolution of 30 minutes or less is sufficient to assess the pathologic tissue's pharmacokineticodynamic curve profile; whereas, in the case of small molecule contrast agents of part hydrophilic character such as serum albumin-associating contrast agent Gadofosveset $(\sim 0.91 \mathrm{kDa})$, which is part neutral and part anionically charged and remains in the blood pool for increased duration [81], as well as in the case of hydrophilic macromolecular contrast and theranostic agents greater than 4 nanometers in diameter, a temporal resolution of upwards of 2 hours is often necessary to properly assess the pathologic tissue's pharmacokineticodynamic curve profile. The visualized contrast concentration results from the combination of contrast-enhanced intra-vascular signal, and in the subset of tissues supplied by blood capillaries permeable to the contrast agent, from the pooling of contrast agent within tissue interstitium followed by intracellular uptake [12], processes that can be separated out by visual inspection of the pathologic tissue's pharmacokineticodynamic curve profile, in context of the blood plasma's pharmacokineticodynamic curve profile, for the theranostic dendrimer, with the potential for increased diagnostic imaging sensitivity upon the assessment of the tissue response pharmacokineticodynamic curve profiles for a couple or series of imageable dendrimer-based contrast agents of various sizes (generations).

The sensitivity of dynamic contrast MRI-based detection of hyperpermeable pathologic tissue utilizing a purely hydrophilic contrast agent is primarily a function of, one, hydrophilic contrast agent size, which increases its blood half-life of the agent, and two, the efficiency by which it relaxes water protons per molar concentration, which increases with polyvalency and then plateaus off. The short blood plasma half-lives of hydrophilic small molecule contrast agents and their lower efficiency to relax water protons (low molar relaxivity) are limitations that can be overcome by decreasing renal filtration fraction [12] or increasing the dose of the contrast agent, both of which increase agent blood plasma half-life, but which also increase the risk for non-selective systemic toxicity of small molecule contrast agents of less than 2 nanometers, which can extravasate appreciably across healthy microvascular barriers, and in the case of small molecule chemotherapy, often becomes the treatment dose-limiting toxicity $[1,2]$. 
In contrast to hydrophilic small molecule contrast agents, the mid-sized higher generation dendrimer-based theranostic agents possess longer blood half lives and those with neutralized-to-anionic exteriors within the optimal size range of 7 to 10 nanometers are passively selective for solid tumor foci and provide exquisite contrast enhancement of solid tumor foci over several hours as they accumulate over time to $0.20 \mathrm{mM}$ average maximum with respect to Gadolinium concentration in peripheral solid tumors and to $0.15 \mathrm{mM}$ average maximum with respect to Gadolinium concentration in intracranial solid tumors $[8,29]$ at significantly lower doses of administration than in their free small molecule form of Gd-DTPA, whereby, the Gd-G5 DTPA-dendrimer (est at $7.5 \mathrm{~nm}$ ) provides superior intra-tumoral accumulation and prolonged contrast enhancement upon intravenous administration at $0.09 \mathrm{mmol} \mathrm{Gd} / \mathrm{kg}$ body weight over 1 minute $[3,8,28,29]$ (Figure 7), as compared to Gd-DTPA administered at the $0.30 \mathrm{mmol} \mathrm{Gd} / \mathrm{kg}$ body weight dose over one minute (triple dose Gd-DTPA), which provides fleeting contrast enhancement [12]. Furthermore, the dosing of Gd-G5 DTPA/DOTA-dendrimers is on the order of $80-100 \mathrm{mg} /$ $\mathrm{kg}$ body weight per dose $[3,8]$ and $1 / 7^{\text {th }}$ to $1 / 2$ of that of hydrophilic small molecule contrast agents such as Gd-DTPA/DOTA administered at triple dose, for which reason the probability for any systemic hypersensitivity associated with MRI imageable dendrimers functionalized biocompatibly with small molecule therapeutics is likely low.

\section{On the applicability of in vitro determinations of} theranostic dendrimer molar relaxivity to the in vivo state The molar relaxivity of the contrast agent, $r\left(\mathrm{~ms}^{-1} / \mathrm{mM}\right)$, which is the amount of increase in the relaxation rate of water protons per unit concentration of agent, has been extensively studied in vitro for a variety of paramagnetic contrast agents at various field strengths $(\mathrm{T})$ at various temperatures $\left({ }^{\circ} \mathrm{C}\right)$ including dendrimer-based, ie $[82,83]$, which has been from the basic science perspective of the physical sciences, with the objective of selecting MRI contrast agents with improved proton longitudinal relaxation efficiency per millimolar concentration of the paramagnetic contrast agent $\left(r_{1}\right.$, .here simply $\left.r\right)$. Based on these in vitro studies, it has been determined that paramagnetic contrast agent molar relaxivity increases with increasing exterior polyvalency of chelated Gadolinium to a point after which it plateaus, whereby the Gd-chelated high-generation dendrimers (Gd-DTPA/DOTA G5 dendrimers and higher) have a plateaued $r$ of $20 \mathrm{~ms}^{-1} / \mathrm{mM}$ at $1.5 \mathrm{~T} @ 20^{\circ}$ [83] and of $\sim 25 \mathrm{~ms}^{-1} / \mathrm{mM}$ at $1.5 \mathrm{~T} @ 37^{\circ}$ $[82,83]$, and a plateaued $r$ of between 10 and $12 \mathrm{~ms}^{-1} / \mathrm{mM}$ at $3.0 \mathrm{~T} @ 37^{\circ}[3,8,28,29,83]$, in contrast to monovalent Gd-DTPA, which has a $r$ of $\sim 4 \mathrm{~ms}^{-1} / \mathrm{mM}$ at $1.5 \mathrm{~T} @ 37^{\circ}$ [81] and a $r$ of $3.8 \mathrm{~ms}^{-1} / \mathrm{mM}$ at $3.0 \mathrm{~T} @ 37^{\circ}$ [12]. These findings are analogous to those noted with respect to the adsorption of several small molecule contrast agents onto nanoporous surfaces [84].

It is difficult, however, to determine the molar relaxivity in vivo as it requires accurate determinations of the actual tissue concentration of the contrast agent at the time of the acquired image, which historically typically has been Gd-DTPA, a hydrophilic contrast agent with a short blood half-life that readily diffuses out of the tissue interstitium $[85,86]$, unlike, for example, $14 \mathrm{C}$-alpha aminoisobutyric acid (14C-AIB) [7,11], a 14C-modified synthetic amino acid that readily localizes intracellularly [7]. In the case of macromoleculars, if the molar relaxivity of a theranostic dendrimer can be determined accurately in vitro, then the in vitro approach would methodologically useful for application across laboratories and centers due to the ease of reproducibility.

From the translational science perspective, it is important to understand the salient biophysical interactions that alter a theranostic agent's molar relaxivity in the physiologic state in vivo. An important observation in this respect is that for a contrast agent with part lipophilic character, the molar relaxivity is much greater in the presence of protein serum albumin, which is the case of Gadofosveset (Vasovist), a Gd-DTPA-based contrast agent of anionic hydro-lipophilic character with serum albumin-associating ability [7] that has a $r$ of $28 \mathrm{~ms}^{-1}$ / $\mathrm{mM}$ at $1.5 \mathrm{~T} @ 37^{\circ}$ [87], in contrast to Gd-DTPA that is of pure hydrophilic character [7] that has a $r$ of $3.8 \mathrm{~ms}^{-1} / \mathrm{mM}$ at $3.0 \mathrm{~T} @ 37^{\circ}$ [12], irrespective of the presence of protein. This observation that there is increased contrast agent molar relaxivity in the presence of associative serum albumins, blood serum and interstitial, is consistent with transiently acquired non-covalent associative polyvalency in the presence of serum albumins, and becomes particularly relevant to take into consideration when designing theranostic dendrimers, as exterior functionalization with moieties such as fluorescent dyes or chemotherapeutics of lipophilic character. In the case of functionalized theranostic dendrimers or other such nanoparticles where foreseeable protein interactions in vivo not considered a priori, the dynamic contrastenhanced MRI-based Gd concentration maps generated on the basis of molar relaxivity determinations of theranostic nanoparticles made in vitro in phosphate buffered saline (PBS) would display greater Gd concentration than actually present intratumorally in the form of Gd-DTPA/DOTA-dendrimer; in the case of theranostic nanoparticles without this exterior introduction of lipophilicity and maintained post-functionalization protruding exterior hydrophilicity (i.e. Gd-DTPAdendrimers), this effect modifier is unlikely to be much of a factor in introducing inaccuracy into the methodology [7]. 
Therefore, the most important realization here is that a functionalized theranostic agent's in vivo molar relaxivity is only equivalent to it's in vivo molar relaxivity if the theranostic agent does not associate with serum proteins: This is only the case for purely hydrophilic theranostic agents of anionically neutralized stable exteriors with covalently bound chelates [2,7]. For theranostic probes without this exterior biophysical character of anionically neutralized exteriors, adjustments would need to be made, and furthermore, the reliability of these adjustments for accurate quantification of theranostic agent concentration in vivo must be questioned.

\section{Methodology for the in vitro determination of theranostic dendrimer molar relaxivity for in vivo application}

Purely hydrophilic theranostic agents of anionically neutralized stable exteriors with covalently bound chelates, the molar relaxivity can be measured accurately in vitro in phosphate buffered saline at body temperature $\left(37^{\circ} \mathrm{C}\right)$ in the $3 \mathrm{~T}$ magnet solenoid coil utilized for the in vivo dynamic contrast-enhanced imaging $[3,8,12,28,29]$, which approximates that of the in vivo state in the absence of any appreciable protein binding, as is the case for pure hydrophiles $[7,88]$. Theranostic dendrimer nanoparticle molar relaxivity is determined by $\mathrm{T}_{1}$-weighted Spin-Echo (SE) MRI methodology utilizing different repetition times $\left(\mathrm{SE} \mathrm{T}_{R}\right.$; ie $100,300,600$ and $1200 \mathrm{~ms}$ ) and a constant echo time ( $\mathrm{SE} \mathrm{T}_{E}$; ie $10 \mathrm{~ms}$ ) for a series of diluted Gd-DTPA-dendrimer PBS solutions with respect to Gadolinium concentration $(0,0.25,0.50,0.75,1.0$ and $2.0 \mathrm{mM}$ ) for the determination of the normalized longitudinal relativity rates $\left(1 / T_{1}\right)$ for each Gadolinium concentration via column ( $\mathrm{m}: \mathrm{T}_{R}$ )-by-row ( $\mathrm{n}$ : Gadolinium concentration) matrix comparisons of contrast-enhanced $\mathrm{T}_{1}$ signal to non-contrast $\mathrm{PBS} \mathrm{T}_{1}$ signal by applying Equation 1:

$$
S=M_{0}\left[1-2 \exp -\left(\frac{\frac{T_{R}-T_{E}}{2}}{T_{1}}\right)+\exp \left(\frac{-T_{R}}{T_{1}}\right)\right]
$$

The Gd-DTPA/DOTA-dendrimer theranostic nanoparticle molar relaxivity rate is calculated by linear regession of normalized longitudinal relativity rates $\left(1 / T_{1}\right)$ plotted on the y-axis versus Gd-DTPA-dendrimer Gadolinium concentration $(0,0.25,0.50,0.75,1.0$ and $2.0 \mathrm{mM})$ plotted on the $\mathrm{x}$-axis, with the slope of this line being the theranostic dendrimer nanoparticle molar relaxivity, $r\left(\mathrm{~ms}^{-1} /\right.$ $\mathrm{mM}$ ), as per the relationship in Equation 2:

$$
r=\frac{\frac{1}{T_{1}}-\frac{1}{T_{10}}}{[\mathrm{C}]}
$$

Since the increase in measured signal intensity is not linear across SE repetition times particularly at concentrations greater than $0.50 \mathrm{mM}$ Gadolinium, imaging of concentration dilutions at multiple repetition times assures accuracy of the in vitro molar relaxivity determination for the entire range of physiologically relevant concentrations of Gadolinium to be encountered in blood plasma $(\sim 1.2 \mathrm{mM})$ and tumor tissue ( 0.1-0.2 mM). The tissue specificity of the spin-echo-based in vitro molar relaxivity determination methodology can be further improved by narrowing the range of in vitro theranostic dendrimer dilutions to that of the Gadolinium concentrations to be encountered specifically in either blood plasma, or in tumor tissue.

The steps for post-processing of the raw Spin Echo molar relaxivity data include molar relaxivity calculations that are performed directly in Matlab following conversion of .Par scan header parameter information into .Brik file signal data and capturing of ROI mask .Brik file signal data into a single Matlab .1D files and then executing UNIX-based custom scripts to batch process, by calling onto the Matlab suite to apply Matlab-based scripts (.m).

\section{Methodology for the dynamic contrast-enhanced magnetic resonance imaging of theranostic dendrimer nanoparticles}

In the case of dynamic contrast-enhanced magnetic resonance imaging, the most applicable methodology for dynamic contrast-enhanced MRI is the $\mathrm{T}_{1}$-weighted SPoiled Gradient Recall Echo (SPGR, GRE; Fast-Field Echo [FFE]) dual-flip angle methodology for quantification of signal intensity in concentration space, which permits the rapid acquisition of high-resolution dynamic scan images over time [80]. Furthermore, SPGR $\mathrm{T}_{1}$-weighted imaging sequences generate homogenous signal for small field-ofviews without tissue interface artifact and are particularly applicable for the treatment cum imaging while delineating discrete volumes of hyperpermeable pathology and pathology-normal tissue interfaces (ie rodent, primate and human tumor foci) following the intravenous bolus administration of paramagnetic theranostic nanoparticles with long blood half lives and low serum albuminassociation potential such as the Gd-DTPA/DOTAdendrimers in the optimal size range of 7 to 10 nanometers.

Being that the maximum achievable magnetization for a radiofrequency pulse is governed by the duration and intensity of the radiofrequency pulse and that a pulse of greater strength yields a larger flip angle $\alpha$, while the $\mathrm{T}_{1^{-}}$ weighted signal decays at a constant rate irrespective of applied radiofrequency energy, the following relationship holds the $\mathrm{T}_{1}$-weighted signal, $S$, in case of the dual flipangle methodology, as defined per Equations 3 and 4:

$$
S=M_{0} \frac{(1-E) \sin \alpha}{1-E \cos \alpha} \quad \text { where } \quad E=\exp \left(-\frac{T_{R}}{T_{1}}\right)
$$




$$
\frac{S_{1}(1-E \cos \alpha 1)}{\sin \alpha 1}=\frac{S_{2}(1-E \cos \alpha 2)}{\sin \alpha 2}
$$

where $\alpha 1=$ low-flip angle for $1^{\text {st }}$ flip radiofrequency pulse signal $S_{1}$ (ie 3 deg).

Where $\alpha 2=$ high=flip angle for $2^{\text {nd }}$ flip radiofrequency pulse signal $\mathrm{S}_{2}$ (ie $12 \mathrm{deg}$ ).

Since the objective is to generate the normalized relative signal pre-contrast map for quantification of contrast agent concentration upon $\alpha 2$ high-flip angle dynamic contrastenhanced image acquisition, Equation 4 can be solved for

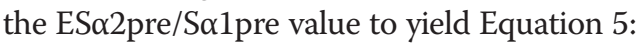

$$
E s_{\alpha} 2 \text { pre } / s_{\alpha} 1 \text { pre }=\frac{S_{2} \sin \alpha 1-S_{1} \sin \alpha 2}{S_{2} \cos \alpha 2 \sin \alpha 1-S_{1} \cos \alpha 1 \sin \alpha 2}
$$

The ES $\alpha 2 p r e / S \alpha 1 p r e$ value is applied to generate the relative normalized $\mathrm{T}_{1}$-weighted signal intensity map as defined per Equation 6:

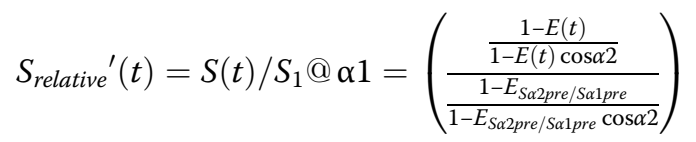

The expression for the decay in electromagnetic energy over time over successive pulses in context of contrast agent-induced tissue contrast enhancement is:

$$
\begin{aligned}
E_{\mathrm{T}_{1 \text { post } / \text { pre }}}(t) & =\exp \left(-\frac{T_{R}}{T_{1 \text { post } / \text { pre }(t)}}\right) \\
& =\exp \left(-\frac{T_{R}}{T_{10 \text { orpre/ } 1 \text { pre }}}-r T_{R} C(t)\right)
\end{aligned}
$$

Knowledge of the relationships defined by Equations 5, 6 and 7 form the basis for quantification of the amount of contrast enhancement at $\alpha 2$ for each image acquisition in concentration space with respect to the imaging agent moiety (i.e. Gadolinium) or even the agent itself (i.e. GdDTPA-dendrimer), where $r$ is the molar relaxivity in $\mathrm{msec}^{-1}$ time/mM contrast agent concentration measured in vitro (Equations 1 and 2) and substituted into Equation 8, whereby the dynamic MRI concentration map is generated voxel-by-voxel $(0.5 \mathrm{~mm}$ by $0.3 \mathrm{~mm}$ by $0.3 \mathrm{~mm})$ per tissue volume slice with respect to contrast agent Gadolinium concentration to quantify the change in the concentration of theranostic dendrimer nanoparticle in the tissue of interest such as blood plasma and solid tumor over time (All figures except Figure 7), which can also be made for a region-ofinterest considered as one large voxel, where applicable (Figure 7). The quantitative dynamic MRI theranostic agent Gd concentration over time is defined as per Equation 8:
For calculation of the change in the agent concentration over time in blood plasma, a few representative voxels are chosen from a small vein or artery, of sufficient diameter including the superior sagittal [venous] sinus (Figure 1) and the common carotid artery (Figure 2): The systemic healthy tissue extraction per circulatory pass in insignificant for optimally sized imageable nanoparticles while the blood transit time from arterial-to-venous circulation is $1 / 4^{\text {th }}$ of that of the temporal resolution $(20 \mathrm{~s}$ per slice) resulting in similar concentrations in either circulation, whereby it is appropriate to utilize SSS voxels for determining blood concentration of the theranostic agent. It is bit more difficult to select artifact-free voxels in the common carotid artery due to flow artifact on slices that are in the periphery of the field-of-view and requires some training. The tumor tissue fractional blood plasma volume $(\mathrm{V} p)$ is relatively insignificant and on the order of 3-5\% and not necessary to factor in for determination of concentrations of interstitial and cellular solid tumor tissue contrast agent concentrations. The concentration of the theranostic dendrimer nanoparticle in the plasma of blood requires knowledge of the red blood cell fraction (Hematocrit: Hct), which is typically around 0.46 in the case of blood-less microsurgery and defined as per Equation 9:

$$
C_{p}=\frac{C_{b}}{1-H c t}
$$

Native acquired dynamic contrast-enhanced MRI signal data are analyzed using the Analysis of Functional NeuroImages (AFNI; http://afni.nimh.nih.gov/) [89] software suite programs such as 3Dvolreg for motion correction, 3Dautobox for cropping, and 3Droistats and 3Dmaskave for extracting parameters for manually-drawn ROI masks in the UNIX programming environment, called-on by simple commands and in custom-made UNIX scripts (.csh). For the dynamic contrast-enhanced MRI signal data, the dual flip angle dynamic contrast-enhanced MRI signal data files (.Par header information and .Rec files) are extracted and converted to native AFNI format signal data (.Head .Brik files), with the entire hi-flip dynamic signal data set motion corrected, after which the low flip and high flip signal data are co-registered, each dynamic high flip angle volume over time to its corresponding low flip angle volume signal data of a single time point, with alignments made by Fourier interpolation. The signal space to concentration space data conversion is then performed by the application of the custom-made UNIX scripts applying Equations 3 through 9.

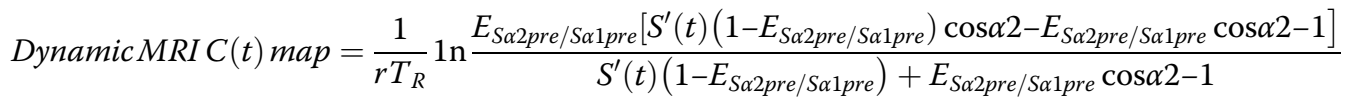




\section{In vivo considerations for theranostic dynamic} contrast-enhanced magnetic resonance imaging

Two different variations of the SPoiled Gradient Recall Echo (SPGR) dual-flip methodology have been applied for in vivo dynamic contrast-enhanced MRI for the quantitative theranostic imaging of functionalized dendrimer nanoparticle accumulation over time into the interstitium of rat glioma-2 (RG-2) malignant gliomas grown orthotopically and ectopically $[3,8,11,12,28,29]$, which are syngenic to the Fischer rat strain $[90,91]$, and equivalent to astrocytomas of the World Health Organization (WHO) Grades III-to-IV (glioblastoma multiforme [GBM]) [55,90,92-94]. In one variation of the methodology, voxel-by-voxel tumor region of interest concentration and maps have been generated via co-registration of slices over time for tumor imaging over 2 hours under continuous general anesthesia sedation without the need for intubation and without complications, which has enabled the systematic study of MRI imageable dendrimer nanoparticle accumulation voxel-byvoxel over time for theranostic purposes [3,8,12,28,29] (Figures 3 through 6; Figure 8); whereas, in the other variation, whole tumor slice volume ROI concentration maps has been performed for determination of change in whole tumor contrast agent concentration over a more prolonged duration of 10 hours upon re-awakening the rodent for 30 minutes in-between 2 minute dynamic scans, also without complications [3,8,29] (Figure 7).

During the course of these laboratory studies, it has been observed that hemodynamic depression resulting from being under continuous sedation results in less MRI imageable dendrimer and theranostic dendrimer nanoparticle accumulation in orthotopic and ectopic solid tumors of rodents (Figures 3 through 6; Figure 8), which is not the case in orthotopic and ectopic solid tumors of rodents awakened in-between dynamic scans (Figure 7), and furthermore, that there is a more significant effect of anesthesia-induced hemodynamic depression on the accumulation of optimally sized MRI imageable dendrimer nanoparticles in the interstitium of extra-cranial peripheral solid tumors as compared to on intra-cranial malignant gliomas, which underscores the effect of hemodynamic depression-induced decreased perfusion on the intratumoral accumulation of even nanoparticle-sized therapeutics in perfusion-limited hyperpermeable pathologies such as peripheral solid tumors with greater permeability surface area product (Ktrans).

\section{Conclusions}

The realization that nanoparticle-based formulations could serve as more efficient molecular forms for either the purpose of imaging, or alternatively, for the purpose of the treatment of disease states, is relatively longstanding in the field of translational nanoparticle science, whereby over the years a variety of functionalized nanoparticles over a spectrum of shapes, sizes and exterior biophysical properties have been investigated for their suitability for either imaging or therapeutic applications, but the majority have failed to meet the criterion of passive selectivity for hyperpermeable pathologies cum biocompatibility in the physiologic state. In more recent years, from 2006 to 2009, quantitative highresolution dynamic contrast-enhanced MRI at a field strength of $3 \mathrm{~T}$ has been utilized for the detailed imaging of the accumulation potential of 2 to 14 nanometer-sized imageable gadolinium chelate-conjugated dendrimer nanoparticles in the solid tumor interstitium of rodent malignant gliomas of volumes as small as $20 \mathrm{~mm}^{3}$, which has been upon bolus intravenous administration over $1 \mathrm{mi}$ nute; based on the findings of this research, it has been discovered that MRI imageable dendrimers are uniquely biocompatible nanoparticles in that optimally designed MRI imageable dendrimers within the 7 to 10 nanometer size range are applicable for the dynamic contrastenhanced imaging and concomitant treatment of malignant solid tumors irrespective of tumor permeability surface area product, and importantly, quantitatively, while affording the ability to detect solid tumor tissue with sensitivity and specificity at the translational level.

The translational quantitative dynamic contrast-enhanced MRI imaging methodology that has been applied, and is discussed herein, utilizes the measure of the ability of a paramagnetic MRI imageable dendrimer functionalized with a small molecule therapeutic to contrast enhance water proton signal, its molar relaxivity $\left(r_{1}\right)$, with the realization that this measure, in fact, can be appropriately determined in vitro utilizing $\mathrm{T}_{1}$-weighted Spin-Echo and applied for the accurate determination of theranostic agent concentration in blood plasma and in the solid tumor tissue interstitium overtime on a voxel-by-voxel basis for an accurate noninvasive assessment of in vivo theranostic pharmacokinetics utilizing $\mathrm{T}_{1}$-weighted Gradient-Recall Echo.

Based on the observations taken together from the findings of these relatively recent research studies, it can be surmised that the optimally functionalized biocompatible theranostic nanoparticle is a non-metal core-based nanoparticle of dendritic architecture in the 7 to 10 nanometer size range with a therapeutic xenobiotic drug covalently bound inwardly via a labile linkage sensitive to $\mathrm{pH}$ (i.e. hydrazone type) along with a covalently bound anionic moiety exteriorly such as an imageable chelate, which imparts dense hydrophilic biophysical character to the exterior. A theranostic nanoparticle based on this design will demonstrate favorable drug release kinetics upon passive selective transvascular passage into the hyperpermeable pathology interstitium during its peak blood plasma concentration, while there being minimal risk for systemic or lymphatic hypersensitivity while in systemic circulation. 
The future perspective is to apply the translational theranostic quantitative dynamic contrast-enhanced MRIbased methodology discussed herein based on knowledge of the optimal nanoparticle size range of 7 to 10 nanometers, density and exterior functionalization necessary for biocompatible passively selective effective transvascular delivery of small molecule therapeutics into solid tumors while maximizing the effectiveness of the approach by locking in MRI imageable theranostic dendrimer nanoparticle treatments for maximum intratumoral accumulation favoring intratumoral release of small molecule drug at peak intratumoral nanoparticle concentration. The further future objective will be to demonstrate the clinical efficacy of the fine tuned refined approach within a few years.

It is foreseeably envisioned that the translational theranostic methodology for diagnostic imaging and the concomitant treatment of solid malignancies discussed herein will result in the effective treatment of solid malignancies, and will also result in the development of effective transvascular therapies for other disease states of pathologic hyperpermeability and without.

\section{Competing Interests}

The author declares that he has no competing interests.

\section{Author's contribution}

HS conceptualized and performed the original research, and wrote the article.

\section{Author's information}

HS: Freelance Investigator in Translational Science and Medicine (unaffiliated).

\section{Acknowledgements}

No funding was acquired for this research. Previous funding entities and authors have been properly acknowledged for their contributions and have been additionally cited herein.

Received: 7 November 2014 Accepted: 21 January 2015 Published online: 21 October 2015

\section{References}

1. Sarin H. Physiologic upper limits of pore size of different blood capillary types and another perspective on the dual pore theory of microvascular permeability. Journal of angiogenesis research. 2010;2:14.

2. Sarin H. Permeation thresholds for hydrophilic small biomolecules across microvascular and epithelial barriers are predictable on the basis of conserved biophysical properties: Pharmacotherapeutic implications. Submitted TBD, TBD(TBD):TBD

3. Sarin H. Recent progress towards development of effective systemic chemotherapy for the treatment of malignant brain tumors. Journal of translational medicine. 2009;7:77.

4. Fung SH, Roccatagliata L, Gonzalez RG, Schaefer PW. MR diffusion imaging in ischemic stroke. Neuroimaging clinics of North America. 2011;21(2):345-77. xi.

5. Habgood MD, Bye N, Dziegielewska KM, Ek CJ, Lane MA, Potter A, et al. Changes in blood-brain barrier permeability to large and small molecules following traumatic brain injury in mice. European Journal of Neuroscience. 2007;25(1):231-8.

6. $G P, J M, C S, J P, M A K, K Q, A S$. Nephrogenic systemic fibrosis: a review of published cases and results from three prospective observational studies. Insights Imaging 2012, 3 (supp 1):S293.

7. Sarin H. Nanomolecular function is conserved in the physiological state. Journal of Nanotechnology: Nanomedicine \& Nanobiotechnology 2015, TBD(TBD):TBD.

8. Essig M, Weber M-A, von Tengg-Kobligk H, Knopp MV, Yuh WTC, Giesel FL. Contrast-Enhanced Magnetic Resonance Imaging of Central Nervous System Tumors: Agents, Mechanisms, and Applications. Topics in Magnetic Resonance Imaging. 2006;17(2):89-106.
9. Sarin $\mathrm{H}$. Effective transvascular delivery of chemotherapy into cancer cells with imageable nanoparticles in the 7 to 10 nanometer size range. In: Current Advances in the Medical Application of Nanotechnology. Volume 1, edn. Bentham Science Publishers Ltd.; 2012: 10-24

10. Tofts PS, Brix G, Buckley DL, Evelhoch JL, Henderson E, Knopp MV, et al. Estimating kinetic parameters from dynamic contrast-enhanced T(1)-weighted MRI of a diffusable tracer: standardized quantities and symbols. J Magn Reson Imaging. 1999;10(3):223-32.

11. Ferrier MC, Sarin H, Fung SH, Schatlo B, Pluta RM, Gupta SN, et al. Validation of dynamic contrast-enhanced magnetic resonance imaging-derived vascular permeability measurements using quantitative autoradiography in the RG2 rat brain tumor model. Neoplasia. 2007;9(7):546-55.

12. Sarin H, Kanevsky AS, Fung SH, Butman JA, Cox RW, Glen D, et al. Metabolically stable bradykinin B2 receptor agonists enhance transvascular drug delivery into malignant brain tumors by increasing drug half-life. Journal of translational medicine. 2009;7:33.

13. Degani H, Gusis V, Weinstein D, Fields S, Strano S. Mapping pathophysiological features of breast tumors by MRI at high spatial resolution. Nature medicine. 1997;3(7):780-2.

14. Tofts PS, Kermode AG. Measurement of the blood-brain barrier permeability and leakage space using dynamic MR imaging. 1. Fundamental concepts. Magn Reson Med. 1991;17(2):357-67.

15. Jackson A, Jayson GC, Li KL, Zhu XP, Checkley DR, Tessier JJ, et al. Reproducibility of quantitative dynamic contrast-enhanced MRI in newly presenting glioma. The British journal of radiology. 2003;76(903):153-62.

16. Padhani AR, Hayes $C$, Landau S, Leach MO. Reproducibility of quantitative dynamic MRI of normal human tissues. NMR in biomedicine. 2002;15(2):143-53.

17. Yang C, Karczmar GS, Medved M, Oto A, Zamora M, Stadler WM. Reproducibility assessment of a multiple reference tissue method for quantitative dynamic contrast enhanced-MRI analysis. Magnetic resonance in medicine: official journal of the Society of Magnetic Resonance in Medicine/ Society of Magnetic Resonance in Medicine. 2009;61(4):851-9.

18. Galbraith SM, Lodge MA, Taylor NJ, Rustin GJ, Bentzen S, Stirling JJ, et al. Reproducibility of dynamic contrast-enhanced MRI in human muscle and tumours: comparison of quantitative and semi-quantitative analysis. NMR in biomedicine. 2002;15(2):132-42.

19. Vincensini D, Dedieu V, Renou JP, Otal P, Joffre F. Measurements of extracellular volume fraction and capillary permeability in tissues using dynamic spin-lattice relaxometry: studies in rabbit muscles. Magnetic resonance imaging. 2003;21(2):85-93.

20. Dedieu V, Bailly C, Vincent C, Achard JL, Le Bouedec G, Penault-Llorca F, et al. Capillary permeability and extracellular volume fraction in uterine cervical cancer as patient outcome predictors: measurements by using dynamic MRI spin-lattice relaxometry. Journal of magnetic resonance imaging: JMRI. 2008;27(4):846-53.

21. Verma S, Turkbey B, Muradyan N, Rajesh A, Cornud F, Haider MA, et al. Overview of dynamic contrast-enhanced MRI in prostate cancer diagnosis and management. AJR American journal of roentgenology. 2012;198(6):1277-88

22. Turnbull LW. Dynamic contrast-enhanced MRI in the diagnosis and management of breast cancer. NMR in biomedicine. 2009;22(1):28-39.

23. Knopp MV, Weiss E, Sinn HP, Mattern J, Junkermann H, Radeleff J, et al. Pathophysiologic basis of contrast enhancement in breast tumors. Journal of magnetic resonance imaging : JMRI. 1999;10(3):260-6.

24. Su MY, Wang Z, Roth GM, Lao X, Samoszuk MK, Nalcioglu O. Pharmacokinetic changes induced by vasomodulators in kidneys, livers, muscles, and implanted tumors in rats as measured by dynamic Gd-DTPA-enhanced MRI. Magn Reson Med. 1996;36(6):868-77.

25. Sarin H. On the future development of optimally-sized lipid-insoluble systemic therapies for CNS solid tumors and other neuropathologies. Recent patents on CNS drug discovery. 2010;5(3):239-52.

26. Sarin $\mathrm{H}$. Overcoming the challenges in the effective delivery of chemotherapies to CNS solid tumors. Therapeutic delivery. 2010;1(2):289-305.

27. de Lussanet QG, Langereis S, Beets-Tan RG, van Genderen MH, Griffioen AW, van Engelshoven JM, et al. Dynamic contrast-enhanced MR imaging kinetic parameters and molecular weight of dendritic contrast agents in tumor angiogenesis in mice. Radiology. 2005;235(1):65-72.

28. Sarin $H$, Kanevsky AS, Wu H, Brimacombe KR, Fung SH, Sousa AA, et al. Effective transvascular delivery of nanoparticles across the blood-brain tumor barrier into malignant glioma cells. Journal of translational medicine. 2008;6:80. 
29. Sarin H, Kanevsky AS, Wu H, Sousa AA, Wilson CM, Aronova MA, et al. Physiologic upper limit of pore size in the blood-tumor barrier of malignant solid tumors. Journal of translational medicine. 2009;7:51.

30. Gerstner ER, Fine RL. Increased permeability of the blood-brain barrier to chemotherapy in metastatic brain tumors: establishing a treatment paradigm. J Clin Oncol. 2007:25(16):2306-12.

31. Neuwelt E, Abbott N, Abrey L, Banks W, Blakley B, Davis T, et al. Strategies to advance translational research into brain barriers. Lancet Neurol. 2008;7:84-96.

32. Kanal E, Maravilla K, Rowley HA. Gadolinium Contrast Agents for CNS Imaging: Current Concepts and Clinical Evidence. AJNR American journal of neuroradiology. 2014;35(12):2215-26.

33. Soo Choi H, Liu W, Misra P, Tanaka E, Zimmer JP, Itty Ipe B, et al. Renal clearance of quantum dots. Nat Biotech. 2007;25(10):1165-70.

34. Louis C, Bazzi R, Marquette CA, Bridot J-L, Roux S, Ledoux G, et al. Nanosized Hybrid Particles with Double Luminescence for Biological Labeling. Chemistry of Materials. 2005;17(7):1673-82.

35. van de Watering FC, Rijpkema M, Perk L, Brinkmann U, Oyen WJ, Boerman OC. Zirconium-89 labeled antibodies: a new tool for molecular imaging in cancer patients. BioMed research international. 2014;2014:203601.

36. Jarrett BR, Frendo M, Vogan J, Louie AY. Size-controlled synthesis of dextran sulfate coated iron oxide nanoparticles for magnetic resonance imaging. Nanotechnology. 2007;18(3):035603.

37. Muldoon L, Sandor M, Pinkston K, Neuwelt E. Imaging, distribution, and toxicity of superparamagnetic iron oxide magnetic resonance nanoparticles in the rat brain and intracerebral tumor. Neurosurgery 2005, 57

38. Moore A, Marecos E, Bogdanov A, Weissleder R. Tumoral distribution of long-circulating dextran-coated iron oxide nanoparticles in a rodent model. Radiology. 2000;214:568-74.

39. Wagner M, Wagner S, Schnorr J, Schellenberger E, Kivelitz D, Krug L, et al. Coronary MR angiography using citrate-coated very small superparamagnetic iron oxide particles as blood-pool contrast agent: initial experience in humans. Journal of magnetic resonance imaging : JMRI. 2011:34(4):816-23.

40. Misselwitz B, Schmitt-Willich H, Ebert W, Frenzel T, Weinmann HJ. Pharmacokinetics of Gadomer-17, a new dendritic magnetic resonance contrast agent. Magma. 2001;12(2-3):128-34.

41. Venditto VJ, Regino CA, Brechbiel MW. PAMAM dendrimer based macromolecules as improved contrast agents. Molecular pharmaceutics. 2005;2(4):302-11.

42. Kobayashi H, Kawamoto S, Sakai Y, Choyke PL, Star RA, Brechbiel MW, et al. Lymphatic drainage imaging of breast cancer in mice by micro-magnetic resonance lymphangiography using a nano-size paramagnetic contrast agent. Journal of the National Cancer Institute. 2004;96(9):703-8.

43. Barrett T, Kobayashi H, Brechbiel M, Choyke PL. Macromolecular MRI contrast agents for imaging tumor angiogenesis. European Journal of Radiology. 2006;60:353-66.

44. Yordanov AT, Kobayashi H, English SJ, Reijnders K, Milenic D, Krishna MC, et al. Gadolinium-labeled dendrimers as biometric nanoprobes to detect vascular permeability. Journal of Materials Chemistry. 2003;13(7):1523-5.

45. Kobayashi H, Reijnders K, English S, Yordanov AT, Milenic DE, Sowers AL, et al. Application of a macromolecular contrast agent for detection of alterations of tumor vessel permeability induced by radiation. Clinical cancer research: an official journal of the American Association for Cancer Research. 2004; 10(22):7712-20.

46. Langer R. Drug delivery and targeting. Nature. 1998;392(6679 Suppl):5-10.

47. Matsumura $Y$, Maeda H. A new concept for macromolecular therapeutics in cancer chemotherapy: Mechanism of tumoritropic accumulation of proteins and the antitumor agent smancs. Cancer Research. 1986;46(12 I):6387.

48. Tomalia DA, Baker H, Dewald J, Hall M, Kallos G, Martin S, et al. A New Class of Polymers: Starburst-Dendritic Macromolecules. Polym J. 1985;17(1):117-32.

49. Brechbiel M, Gansow O, Atcher R, Schlom J, Esteban J, Simpson D, et al. Synthesis of 1-(p-isothiocyanatobenzyl) derivatives of DTPA and EDTA. Antibody labeling and tumor-imaging studies. Inorganic Chemistry. 1986;25:2772-81.

50. Maeda $\mathrm{H}$. Vascular permeability in cancer and infection as related to macromolecular drug delivery, with emphasis on the EPR effect for tumor-selective drug targeting. Proceedings of the Japan Academy Series B, Physical and biological sciences. 2012;88(3):53-71.

51. Rosier RN, O'Keefe RJ, Teot LA, Fox EJ, Nester TA, Puzas JE, et al. P-glycoprotein expression in cartilaginous tumors. Journal of surgical oncology. 1997:65(2):95-105.

52. Brem H, Mahaley M, Vick N, Black K, Schold S, Burger $P$, et al. Interstitial chemotherapy with drug polymer implants for the treatment of recurrent gliomas. J Neurosurg. 1991;74:441-6.
53. Campbell RB, Fukumura D, Brown EB, Mazzola LM, Izumi Y, Jain RK, et al. Cationic charge determines the distribution of liposomes between the vascular and extravascular compartments of tumors. Cancer research. 2002;62(23):6831-6.

54. Stylianopoulos T, Soteriou K, Fukumura D, Jain R. Cationic Nanoparticles Have Superior Transvascular Flux into Solid Tumors: Insights from a Mathematical Model. Ann Biomed Eng. 2013;41(1):68-77.

55. Hobbs SK, Monsky WL, Yuan F, Roberts WG, Griffith L, Torchilin VP, et al. Regulation of transport pathways in tumor vessels: role of tumor type and microenvironment. Proc Natl Acad Sci U S A. 1998;95(8):4607-12.

56. Chauhan VP, Stylianopoulos T, Martin JD, Popovic Z, Chen O, Kamoun WS, et al. Normalization of tumour blood vessels improves the delivery of nanomedicines in a size-dependent manner. Nature nanotechnology. 2012;7(6):383-8.

57. Mross K, Niemann B, Massing U, Drevs J, Unger C, Bhamra R, et al. Pharmacokinetics of liposomal doxorubicin (TLC-D99; Myocet) in patients with solid tumors: an open-label, single-dose study. Cancer Chemother Pharmacol. 2004;54(6):514-24.

58. Lotem M, Hubert A, Lyass $\mathrm{O}$, Goldenhersh MA, Ingber A, Peretz $T$, et al. Skin toxic effects of polyethylene glycol-coated liposomal doxorubicin. Archives of Dermatology. 2000;136(12):1475-80.

59. Taupitz M, Schnorr J, Abramjuk C, Wagner S, Pilgrimm H, Hunigen $\mathrm{H}$, et al. New generation of monomer-stabilized very small superparamagnetic iron oxide particles (VSOP) as contrast medium for MR angiography: preclinical results in rats and rabbits. Journal of magnetic resonance imaging: JMRI. 2000;12(6):905-11.

60. Jackson C, Chanzy H, Booy F, Drake B, Tomalia D, Bauer B, et al. Visualization of dendrimer molecules by transmission electron microscopy (TEM): Staining methods and cryo-TEM of vitrified solutions. Macromolecules. 1998;31:6259-65.

61. Tomalia DA, Frechet JM. Discovery of dendrimers and dendritic polymers: a brief historical perspective. Journal of Polymer Science, Part A: Polymer Chemistry. 2002;40(16):2719-28.

62. Tomalia DA. Dendrons/dendrimers. The convergence of quantized dendritic building blocks/architectures for applications in nanotechnology. Chimica Oggi. 2005;23(6):41.

63. Gillies ER, Dy E, Frechet JMJ, Szoka FC. Biological evaluation of polyester dendrimer: Poly(ethylene oxide) "bow-tie" hybrids with tunable molecular weight and architecture. Molecular Pharmaceutics. 2005;2(2):129.

64. Lee CC, Gillies ER, Fox ME, Guillaudeu SJ, Frechet JMJ, Dy EE, et al. A single dose of doxorubicin-functionalized bow-tie dendrimer cures mice bearing C-26 colon carcinomas. Proceedings of the National Academy of Sciences of the United States of America. 2006;103(45):16649.

65. Kono K, Kojima C, Hayashi N, Nishisaka E, Kiura K, Watarai S, et al. Preparation and cytotoxic activity of poly(ethylene glycol)-modified poly(amidoamine) dendrimers bearing adriamycin. Biomaterials. 2008;29(11):1664-75

66. Choi Y, Baker Jr JR. Targeting cancer cells with DNA-assembled dendrimers: a mix and match strategy for cancer. Cell cycle. 2005;4(5):669-71.

67. Tekade RK, Dutta T, Tyagi A, Bharti AC, Das BC, Jain NK. Surface-engineered dendrimers for dual drug delivery: a receptor up-regulation and enhanced cancer targeting strategy. Journal of drug targeting. 2008;16(10):758-72.

68. Okuda T, Kawakami S, Akimoto N, Niidome T, Yamashita F, Hashida M. PEGylated lysine dendrimers for tumor-selective targeting after intravenous injection in tumor-bearing mice. J Control Release. 2006;116(3):330-6.

69. Kojima C, Regino CA, Umeda Y, Kobayashi H, Kono K. Influence of dendrimer generation and polyethylene glycol length on the biodistribution of PEGylated dendrimers. International Journal of Pharmaceutics. 2010;383:293-6.

70. Kukowska-Latallo JF, Candido KA, Cao Z, Nigavekar SS, Majoros IJ, Thomas TP, et al. Nanoparticle targeting of anticancer drug improves therapeutic response in animal model of human epithelial cancer. Cancer Research. 2005;65(12):5317.

71. Swanson SD, Kukowska-Latallo JF, Patri AK, Chen C, Ge S, Cao Z, et al. Targeted gadolinium-loaded dendrimer nanoparticles for tumor-specific magnetic resonance contrast enhancement. International Journal of Nanomedicine. 2008;3(2):201.

72. Thomas TP, Huang B, Choi SK, Silpe JE, Kotlyar A, Desai AM, et al. Polyvalent dendrimer-methotrexate as a folate receptor-targeted cancer therapeutic. Molecular pharmaceutics. 2012;9(9):2669-76.

73. Konda SD, Wang S, Brechbiel M, Wiener EC. Biodistribution of a 153 Gd-folate dendrimer, generation $=4$, in mice with folate-receptor positive and negative ovarian tumor xenografts. Investigative radiology. 2002;37(4):199-204. 
74. Li MH, Choi SK, Thomas TP, Desai A, Lee KH, Kotlyar A, et al. Dendrimer-based multivalent methotrexates as dual acting nanoconjugates for cancer cell targeting. Eur J Med Chem. 2012;47(1):560-72.

75. Sousa AA, Aronova MA, Wu H, Sarin H, Griffiths GL, Leapman RD. Determining molecular mass distributions and compositions of functionalized dendrimer nanoparticles. Nanomedicine. 2009;4(4):391-9.

76. Sousa A, Aronova M, Wu H, Sarin H, Griffiths G, Leapman R. Quantitative STEM and EFTEM characterization of dendrimer-based nanoparticles used in magnetic resonance imaging and drug delivery. Microsc Microanal. 2008;14 Suppl 2:694-5.

77. Lee CC, Gillies ER, Fox ME, Guillaudeu SJ, Frechet JM, Dy EE, et al. A single dose of doxorubicin-functionalized bow-tie dendrimer cures mice bearing C-26 colon carcinomas. Proc Natl Acad Sci U S A. 2006;103(45):16649-54.

78. Swanson SD, Kukowska-Latallo JF, Patri AK, Chen C, Ge S, Cao Z, et al. Targeted gadolinium-loaded dendrimer nanoparticles for tumor-specific magnetic resonance contrast enhancement. International journal of nanomedicine. 2008;3(2):201-10.

79. Yue Y, Eun JS, Lee MK, Seo SY. Synthesis and characterization of G5 PAMAM dendrimer containing daunorubicin for targeting cancer cells. Archives of pharmacal research. 2012;35(2):343-9.

80. Haacke EM, Brown RW, Thompson MR, Venkatesan M. Magnetic Resonance Imaging: Physical Principles and Sequence Design. New York: Wiley; 1999.

81. Aime S, Caravan P. Biodistribution of gadolinium-based contrast agents, including gadolinium deposition. Journal of magnetic resonance imaging: JMRI. 2009;30(6):1259-67.

82. Bryant Jr LH, Brechbiel MW, Wu C, Bulte JW, Herynek V, Frank JA. Synthesis and relaxometry of high-generation $(G=5,7,9$, and 10) PAMAM dendrimer-DOTA-gadolinium chelates. Journal of magnetic resonance imaging: JMRI. 1999;9(2):348-52.

83. Langereis S, de Lussanet QG, van Genderen MH, Meijer EW, Beets-Tan RG, Griffioen AW, et al. Evaluation of Gd(III)DTPA-terminated poly(propylene imine) dendrimers as contrast agents for MR imaging. NMR in biomedicine. 2006;19(1):133-41.

84. Sethi R, Ananta JS, Karmonik C, Zhong M, Fung SH, Liu X, et al. Enhanced MRI relaxivity of $\mathrm{Gd}(3+)$-based contrast agents geometrically confined within porous nanoconstructs. Contrast media \& molecular imaging. 2012;7(6):501-8.

85. Orth RC, Bankson J, Price R, Jackson EF. Comparison of single- and dual-tracer pharmacokinetic modeling of dynamic contrast-enhanced MRI data using low, medium, and high molecular weight contrast agents. Magnetic resonance in medicine: official journal of the Society of Magnetic Resonance in Medicine/ Society of Magnetic Resonance in Medicine. 2007;58(4):705-16.

86. Landis CS, Li X, Telang FW, Coderre JA, Micca PL, Rooney WD, et al. Determination of the MRI contrast agent concentration time course in vivo following bolus injection: Effect of equilibrium transcytolemmal water exchange. Magnetic Resonance in Medicine. 2000;44(4):563-74.

87. Eldredge HB, Spiller M, Chasse JM, Greenwood MT, Caravan P. Species dependence on plasma protein binding and relaxivity of the gadolinium-based MRI contrast agent MS-325. Investigative radiology. 2006;41(3):229-43.

88. Donahue KM, Burstein D, Manning WJ, Gray ML. Studies of Gd-DTPA relaxivity and proton exchange rates in tissue. Magn Reson Med. 1994;32(1):66-76.

89. Cox RW. AFNI: software for analysis and visualization of functional magnetic resonance neuroimages. Comput Biomed Res. 1996;29(3):162-73.

90. Aas AT, Brun A, Blennow C, Stromblad S, Salford LG. The RG2 rat glioma model. J Neurooncol. 1995;23(3):175-83.

91. Barth R. Rat brain tumor models in experimental neuro-oncology: The $9 \mathrm{~L}$, C6, T9, F98, RG2 (D74), RT-2 and CNS-1 gliomas. Journal of Neuro-Oncology. 1998;36:91-102.

92. Camphausen K, Purow B, Sproull M, Scott T, Ozawa T, Deen DF, et al. Influence of in vivo growth on human glioma cell line gene expression: convergent profiles under orthotopic conditions. Proc Natl Acad Sci U S A. 2005;102(23):8287-92.

93. Wen PY, Kesari S. Malignant Gliomas in Adults. New England Journal of Medicine. 2008;359(5):492-507.

94. Kleinschmidt-DeMasters BK, Lillehei KO, Breeze RE. Neoplasms involving the central nervous system in the older old. Human pathology. 2003:34(11):1137-47.

\section{Submit your next manuscript to BioMed Central and take full advantage of:}

- Convenient online submission

- Thorough peer review

- No space constraints or color figure charges

- Immediate publication on acceptance

- Inclusion in PubMed, CAS, Scopus and Google Scholar

- Research which is freely available for redistribution

Submit your manuscript at www.biomedcentral.com/submit 